\title{
Endothelial chimerism and vascular sequestration protect pancreatic islet grafts from antibody-mediated rejection
}

\author{
Chien-Chia Chen, ${ }^{1}$ Eric Pouliquen, ${ }^{2}$ Alexis Broisat, ${ }^{3}$ Francesco Andreata, ${ }^{4}$ Maud Racapé, ${ }^{5}$ Patrick Bruneval, ${ }^{5}$ Laurence Kessler, ${ }^{6,7}$ \\ Mitra Ahmadi, ${ }^{3}$ Sandrine Bacot, ${ }^{3}$ Carole Saison-Delaplace, ${ }^{1,2}$ Marina Marcaud, ${ }^{2}$ Jean-Paul Duong Van Huyen, ${ }^{5}$ Alexandre Loupy, ${ }^{5,8}$ \\ Jean Villard, ${ }^{9}$ Sandrine Demuylder-Mischler, ${ }^{10}$ Thierry Berney, ${ }^{7,10}$ Emmanuel Morelon, ${ }^{1,2,711}$ Meng-Kun Tsai, ${ }^{12}$ \\ Marie-Nathalie Kolopp-Sarda, ${ }^{13}$ Alice Koenig, ${ }^{1}$ Virginie Mathias, ${ }^{14}$ Stéphanie Ducreux, ${ }^{14}$ Catherine Chezzi, ${ }^{3}$ \\ Valerie Dubois, ${ }^{14}$ Antonino Nicoletti, ${ }^{4}$ Thierry Defrance, ${ }^{1}$ and Olivier Thaunat ${ }^{1,2,7,11}$ \\ ${ }^{1}$ French National Institute of Health and Medical Research (INSERM) Unit 1111, Lyon, France. ${ }^{2}$ Edouard Herriot University Hospital, Department of Transplantation, Nephrology and Clinical Immunology, \\ Lyon, France. ${ }^{3}$ French National Institute of Health and Medical Research (INSERM) Unit 1039, Grenoble, France; Bioclinical Radiopharmaceutical Laboratory, Joseph Fourier University (Grenoble 1), \\ Grenoble, France. ${ }^{4}$ French National Institute of Health and Medical Research (INSERM) Unit 1148, Laboratory of Vascular Translational Science, F-75018, Paris, France; Paris Diderot University, Paris, France. \\ ${ }^{5}$ Paris Translational Research Centre for Organ Transplantation, Paris Descartes University, Paris, France. ${ }^{6}$ Department of Diabetology, University Hospital, Strasbourg, France; Federation of Translational \\ Medicine of Strasbourg, University of Strasbourg, Strasbourg, France. ${ }^{7}$ Groupe Rhin-Rhône-Alpes-Cenève pour la Greffe d'llots de Langerhans (GRAGIL) Consortium. ${ }^{8}$ Department of Kidney Transplantation, \\ Necker Hospital, Assistance Publique-Hôpitaux de Paris, Paris, France. ̊Department of Immunology and Allergy and Department of Laboratory Medicine, Geneva University Hospital, Geneva, Switzerland. \\ ${ }^{10}$ Department of Surgery, Islet Isolation, and Transplantation Center, Geneva University Hospitals, Geneva, Switzerland. "'Lyon-Est Medical Faculty, Claude Bernard University (Lyon 1), Lyon, France. \\ ${ }^{12}$ Department of Surgery, National Taiwan University Hospital and National Taiwan University, College of Medicine, Taipei, Taiwan. ${ }^{33}$ Lyon-Sud University Hospital, Laboratory of Immunology, Lyon, France. \\ ${ }^{14}$ French National Blood Service (EFS), HLA Laboratory, Lyon, France.
}

Humoral rejection is the most common cause of solid organ transplant failure. Here, we evaluated a cohort of 49 patients who were successfully grafted with allogenic islets and determined that the appearance of donor-specific anti-HLA antibodies (DSAs) did not accelerate the rate of islet graft attrition, suggesting resistance to humoral rejection. Murine DSAs bound to allogeneic targets expressed by islet cells and induced their destruction in vitro; however, passive transfer of the same DSAs did not affect islet graft survival in murine models. Live imaging revealed that DSAs were sequestrated in the circulation of the recipients and failed to reach the endocrine cells of grafted islets. We used murine heart transplantation models to confirm that endothelial cells were the only accessible targets for DSAs, which induced the development of typical microvascular lesions in allogeneic transplants. In contrast, the vasculature of DSA-exposed allogeneic islet grafts was devoid of lesions because sprouting of recipient capillaries reestablished blood flow in grafted islets. Thus, we conclude that endothelial chimerism combined with vascular sequestration of DSAs protects islet grafts from humoral rejection. The reduced immunoglobulin concentrations in the interstitial tissue, confirmed in patients, may have important implications for biotherapies such as vaccines and monoclonal antibodies.

\section{Introduction}

Type 1 diabetes is one of the most prevalent chronic diseases of childhood. Epidemiologic studies have estimated that approximately 70,000 new cases are diagnosed every year and that the incidence of the disease is increasing annually by $2 \%-5 \%$ worldwide (1).

Type 1 diabetes is caused by an autoimmune-mediated destruction of pancreatic $\beta$ cells, one of the 4 major cell types present in the islets of Langerhans $(65 \%-80 \%$ of the cells). $\beta$ Cells are unique cells in the pancreas that synthesize and secrete insulin, a key hormone for glucose level regulation. $\beta$ Cell destruction therefore results in life-long dependence on exogenous insulin supply. Despite advances in insulin formulations, insulin-delivery systems, and glucose-monitoring devices/strategies, less than

Conflict of interest: The authors have declared that no conflict of interest exists Submitted: February 23, 2017; Accepted: October 12, 2017. Reference information: J Clin Invest. 2018;128(1):219-232. https://doi.org/10.1172/JCI93542. one-third of patients meet clinical care targets needed to prevent secondary end-organ complications such as retinal, renal, and neurological diseases (2).

Early rodent experiments have demonstrated that islets of Langerhans can be isolated from pancreas exocrine component and grafted into diabetic mice (3). Advances in pancreatic islet isolation and refinement of immunosuppressive protocols allowed a first patient to achieve insulin independence in 1990 (4). In contrast to intensive insulin regimens and insulin pumps, pancreatic islet grafting can restore endogenous insulin secretion, which efficiently prevents, halts, or reverses the development or progression of secondary diabetes complications $(5,6)$. Unfortunately, islet function decreases over time such that by 3 years after transplant, less than $50 \%$ of the recipients remain insulin independent (7).

Identifying the causes of progressive attrition of grafted islets appears to be a critical step in improving long-term results. The recipient's immune system is likely involved in this process, since the graft function is more resilient in autografts than allografts (8). Alloim- 

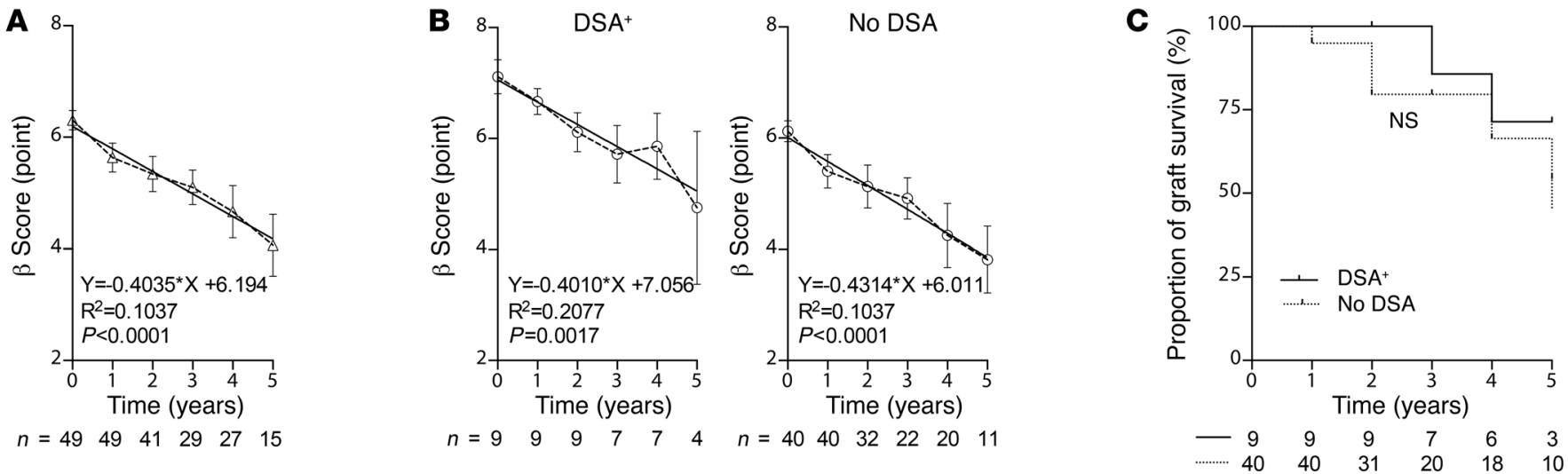

Figure 1. DSA did not affect pancreatic islet graft function. Pancreatic islet graft function of 49 patients was assessed every year using the $\beta$ score (mean \pm SD). Linear regression was used to estimate the relation between time and pancreatic islet graft function. (A) The regression line slope indicates the rate of islet graft attrition in the cohort. (B) Nine patients developed de novo donor-specific anti-HLA Abs (DSA), all in the first year after grafting. The rate of pancreatic islet graft attrition was estimated for the 9 patients with DSA (left, DSA+) and the remaining 40 patients without DSA (right, No DSA). (C) Islet graft survival curves for recipients on immunosuppression with (solid line) or without DSA (dashed line) are compared. NS, $P=0.3367$, log-rank test.

mune response drives rejection through 2 distinct effector arms: Abs and cytotoxic T cells. Donor-specific anti-HLA antibodies (DSAs) are increasingly recognized as the prime cause of solid organ transplant failure $(9,10)$. As with solid organ recipients, islet allograft recipients also develop DSA (11-13); however, the impact of DSA on islet graft function remains puzzling. Indeed, the few studies that have investigated whether DSA generation correlated with decreased islet graft survival have reached opposite conclusions (11-14).

Here, we demonstrate that allogeneic islets are resistant to DSA-mediated rejection despite the fact that DSA can destroy islet cells in vitro. Using murine experimental models, we show that the resistance of allogeneic islets to humoral rejection is explained by the combination of (i) vascular sequestration of DSAs, which are unable to access the allogeneic $\beta$ cells in vivo, and (ii) the fact that unlike vascularization of transplanted organs (that comes from the donor), islet graft vascularization develops from the recipient and hence is not targeted by DSA.

\section{Results}

DSAs do not accelerate pancreatic islet graft attrition in the clinic. In order to evaluate the impact of DSA on islet graft survival, we undertook a retrospective multicentric study. The medical files of all patients successfully grafted with allogeneic islets within the GRAGIL consortium between 2000 and 2016 were reviewed. Forty-nine recipients had sufficient available clinical and biological material to be retrospectively analyzed (see the characteristics of the study population in Supplemental Table 1; supplemental material available online with this article; https://doi.org/10.1172/JCI93542DS1).

The $\beta$ score (15), which integrates insulin requirements, C-peptide secretion, and glycemic control, was used to monitor the pancreatic islet graft function of the cohort longitudinally. As expected, $\beta$ cell function decreased regularly over the follow-up period, as reflected by the slope of linear regression $(-0.4 \beta$ score points per year; Figure 1A).

Among these 49 patients, 9 patients with functional islet grafts developed DSA while on immunosuppressive therapy. All 9 recipients developed DSA (median of the mean fluorescence intensity [MFI]: 2,860; range: 700-8,900) in the first year after grafting, mainly against mismatched HLA II molecules (6/9, $66 \%)$. Interestingly, the rate of islet graft attrition of the 9 recipients with DSA was remarkably similar to the rate of islet graft attrition of patients without DSA (DSA vs. no DSA: -0.40 vs. $-0.43, \beta$ score point per year; Figure $1 \mathrm{~B})$, as were graft survivals of the 2 groups (Figure $1 \mathrm{C}$ ).

Although the correlative nature of the study did not allow for drawing definitive conclusions, these results suggest that, in contrast with solid organ transplants, grafted islets are resistant to humoral rejection, since the kinetics of graft loss would otherwise have been faster.

Murine model of intrahepatic pancreatic islet grafting recapitulates the clinical findings in patients with DSA. We therefore next used a mouse model to explore how pancreatic islet would be preserved despite the presence of DSA. WT C57BL/6 $\left(\mathrm{H}-2^{\mathrm{b}}\right)$ mice were rendered diabetic by administration of i.p. injection of streptozotocin. Diabetic mice were used as recipients of purified pancreatic islets (Figure 2A), which were then injected in the portal vein to mimic the grafting procedure used in the clinic (Figure $2 \mathrm{~B}$ ). Pancreatic islet morphology was assessed in the liver of syngeneic recipient mice 50 days after grafting. Morphologically intact islets were found scattered within hepatic parenchyma, close to the blood vessels of the portal tract (Figure 2C).

The grafted mice normalized their glycemia within 4 days (Figure 2D). WT C57BL/6 $\left(\mathrm{H}-2^{\mathrm{b}}\right)$ mice tolerated the syngeneic islet grafts, since they remained euglycemic until the end of follow-up (Figure 2D). Islets from CBA mice $\left(\mathrm{H}-2^{\mathrm{k}}\right)$ triggered an allogeneic response in the recipient mice, as reflected by the generation of DSAs, which were detected in the recipients' circulation and could be quantified using a customized flow cross-match assay (Supplemental Figure 1 and Figure 2E). The recipient's allogeneic response led to the destruction of grafted islets, as shown by a return to hyperglycemia within 15 days (syngeneic vs. allogeneic islet survival, $P=0.0008$, log-rank test; Figure 2D). Consistent with what was observed in the clinic, islet graft survival was similar for recipient mice with a DSA titer above versus below the median value ( $P=0.27$, log-rank test). 
$\mathbf{A}$

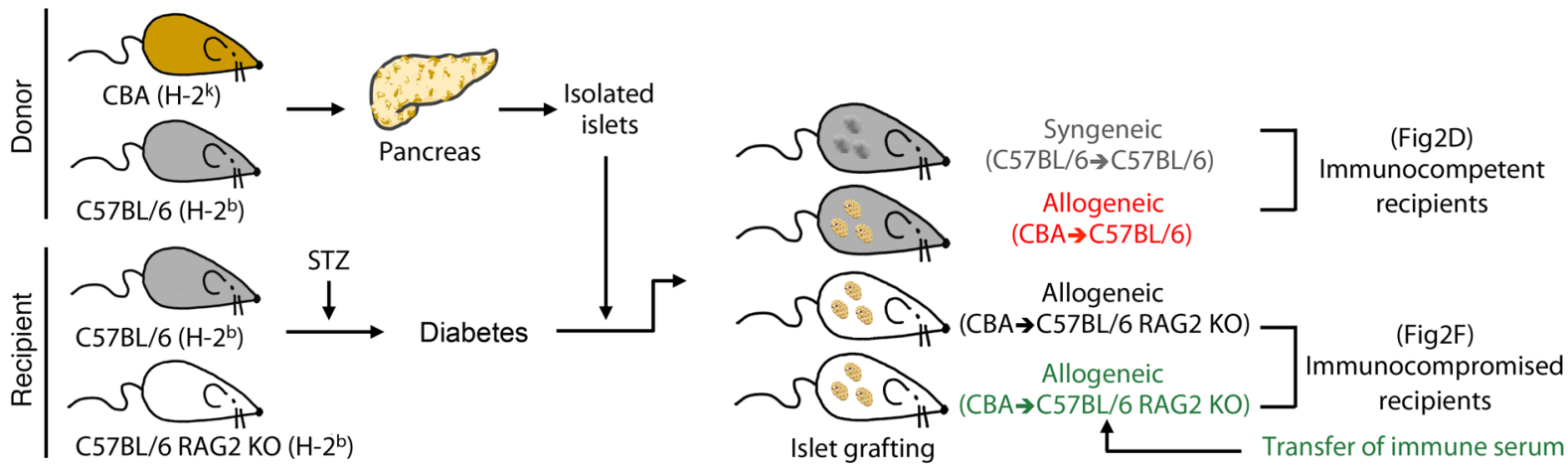

B
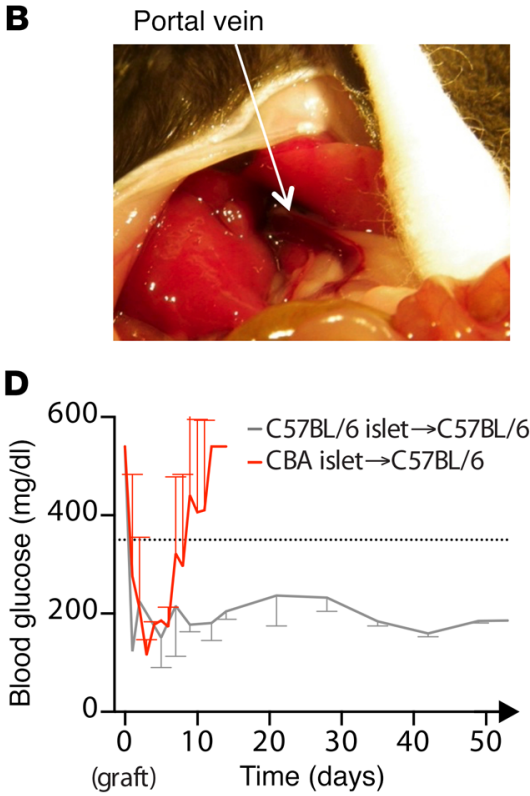

C
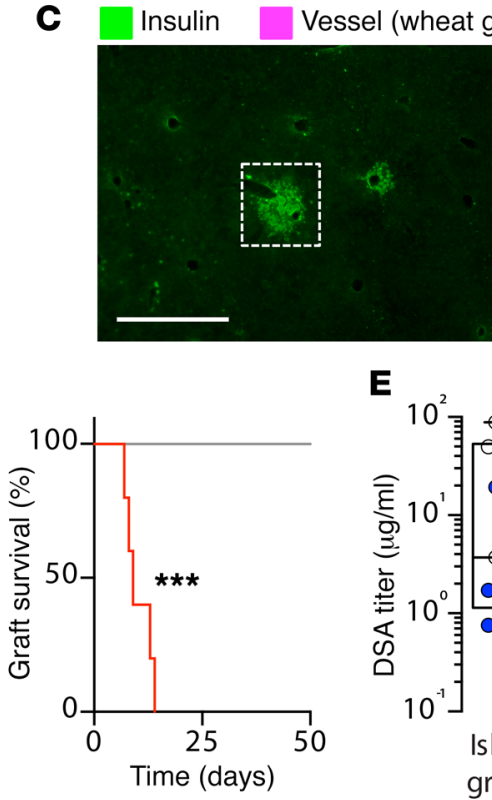

E

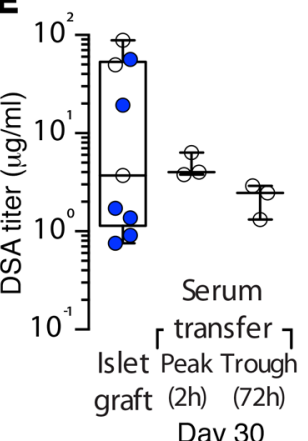

$\mathbf{F}$

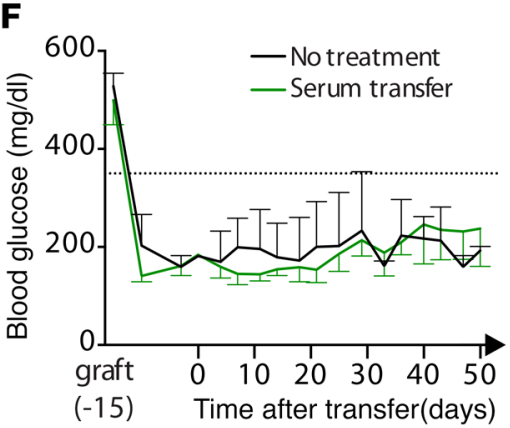

G

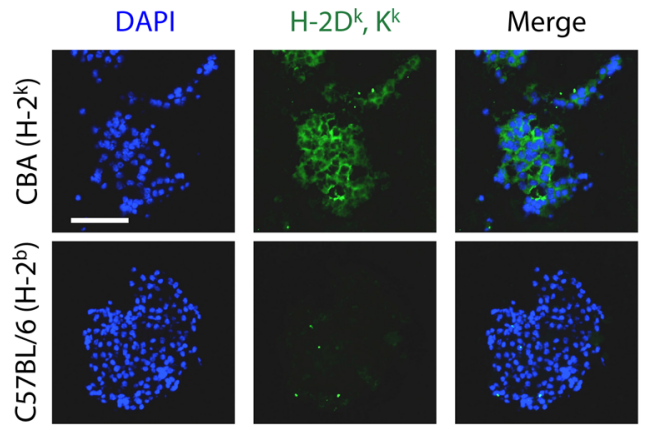

H

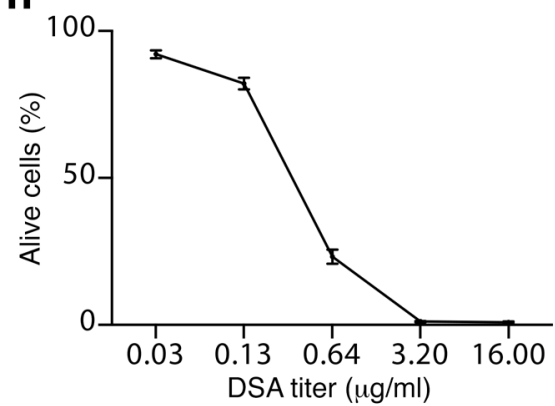

I

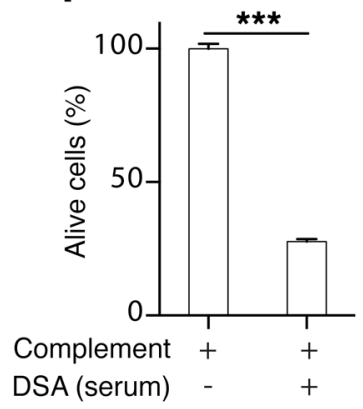

Figure 2. Experimental model recapitulates clinical findings. (A) Schematic of different experimental models of pancreas islet grafting. (B) View of operative site exposing recipient portal vein (white arrow), in which purified islets were injected. (C) Left: representative finding of immunofluorescence analyses performed 50 days after intraportal injection of syngeneic islets. Right: magnification of the white square shown on thumbnail. Scale bars: $500 \mu \mathrm{m}$ (left); $100 \mu \mathrm{m}$ (right). (D) Left: blood glucose level was measured twice weekly in WT C57BL/6 recipients. Evolution of glycemia (mean \pm SD) is shown for C57BL/6 (syngeneic, gray; $n=2$ ) and CBA (allogeneic, red; $n=5$ ) grafts. Islet graft loss was defined by fasting glycemia of more than $350 \mathrm{mg} / \mathrm{dl}$ (dashed line). Right: survival curves for C57BL/6 (syngeneic, gray; $n=2$ ) and CBA (allogeneic, red; $n=5$ ) grafts. ${ }^{* * *} P=0.0008$, log-rank test. (E) Flow cytometry cross-match technique was used to quantify circulating DSA generated by WT C57BL/6 recipients in response to intraportal CBA islet graft. Individual values measured at peak of humoral alloimmune response of 2 independent experiments (white and blue symbols) are shown. The same technique was applied to monitor peak and trough levels of circulating DSA 30 days after starting passive i.v. transfer of immune serum. (F) Blood glucose levels were measured twice weekly in C57BL/6 RAG2 KO recipients of an intraportal CBA islet graft. Evolution of glycemia (mean $\pm \mathrm{SD}$ ) is shown for recipients transferred (green; $n=4$ ) or not (black; $n=5$ ) with immune serum. (C) H-2k expression was assessed on CBA ( $\mathrm{H}-2^{\mathrm{k}}$, upper row) and C57BL/6 ( $\mathrm{H}-2^{\mathrm{b}}$, lower row) freshly isolated pancreatic islets. Scale bar: $100 \mu \mathrm{m}$. (H and I) Cytotoxic potential of immune serum was evaluated in vitro using complement-dependent cytotoxic assay (mean $\pm \mathrm{SD}$ ) on CBA splenocytes (H) and CBA pancreatic islet cell suspension (I). ${ }^{* *} P<0.001,1$-way ANOVA. 
In contrast with WT animals, C57BL/6 RAG2 KO mice, which lack $\mathrm{T}$ and $\mathrm{B}$ cells but have a functional innate immune system, did not reject CBA islet grafts (Figure 2F). These results highlight the central role of adaptive immunity in islet graft rejection and indicate that this model could be used to separately evaluate the impact of the 2 effector arms of the adaptive immune system, i.e., Abs and T cells.

In line with the latter idea, purified $\mathrm{T}$ cells were first transferred to C57BL/6 RAG2 KO recipients, which led to prompt rejection of allogeneic CBA islets, despite the absence of DSA (Supplemental Figure 2). These data established that the $\mathrm{T}$ cell-mediated response is sufficient to reject an allogeneic islet graft.

The impact of the sole recipient's humoral alloimmune response on islet graft survival was then evaluated using passive transfer of DSA. Immune sera were collected from WT C57BL/6 recipients $\left(\mathrm{H}-2^{\mathrm{b}}\right)$ during the primary immune response against $\mathrm{H}-2^{\mathrm{k}}$ alloantigens (for details, see Supplemental Methods and Supplemental Figure 3, A-E). Infusions (i.v.) of immune sera were started 15 days after the grafting procedure to mimic the clinical situation of patients developing de novo DSA. The amount of immune sera infused was set to match the DSA peak, with the median value of DSA concentration observed in WT C57BL/6 islet recipients (Figure $2 \mathrm{E}$ ). The frequency of immune sera infusions (every 72 hours) was set to keep the titer of circulating DSA close to the median value of the DSA concentration observed in WT C57BL/6 islet recipients (Figure 2E). In contrast with recipients transferred with purified T cells, mice transferred with DSA maintained normal glycemia during the follow-up period (Figure 2F) despite the fact that the grafted islets did express $\mathrm{H}-2$ molecules of the donor haplotype $\left(\mathrm{H}-2^{\mathrm{k}}\right)$ (Figure $\left.2 \mathrm{G}\right)$. This finding is reminiscent of our observation made in the clinic and again raised the question of why grafted islets were resistant to DSA-mediated destruction.

Recipients' humoral response destroys allogeneic islets in vitro. A first possibility to explain the lack of a deleterious impact of DSA on grafted islets is that the recipient's humoral response might not be of sufficient quantity and/or quality. Indeed, beyond the mere quantity (i.e., the titer) of DSA, several recent clinical studies have highlighted the importance of the "quality" of DSA, i.e., avidity, nature of heavy chain isotypes (16), and ability to activate the complement cascade $(17,18)$.

To test this hypothesis, DSAs generated by WT C57BL/6 recipients of a CBA islet graft were tested in vitro. The ability of sensitized sera to destroy allogeneic cellular targets was assessed in a complement-dependent lymphocytotoxicity test (Figure $2 \mathrm{H}$ ). Of note, a DSA titer of $3.2 \mu \mathrm{g} / \mathrm{ml}$ (equivalent to the median value observed in WT C57BL/6 islet recipients and used in transfer experiments, Figure 2E) was sufficient to destroy $100 \%$ of the allogeneic lymphocytes in vitro (Figure 2H). This DSA titer was also sufficient to promote the in vitro destruction of $75 \%$ of the allogeneic islet cells used as targets (Figure 2I).

To determine whether increasing DSA avidity would restore their deleterious impact in vivo, we compared the function of CBA islets grafted in C57BL/6 RAG2 KO recipients that were passively transferred with immune sera collected either during primary (low-avidity DSA) or memory (high-avidity DSA) alloimmune responses (for details, see Supplemental Methods and Supplemental Figure 3C). Both groups of mice maintained normal glycemia during all of the follow-up period, demonstrating that increasing
DSA avidity is not sufficient to restore the toxicity on allogeneic grafts in vivo (Supplemental Figure 3F).

Together, these results argued against the hypothesis of a defect in the alloimmune humoral response generated by islet graft recipients. Instead, they suggest that the resistance of allogeneic islets to DSA-mediated destruction in vivo may be due to graft-intrinsic properties.

Refining the experimental model to decipher the mechanisms of islet graft resistance to DSA-mediated rejection in vivo. Intraportal injection of pancreatic islets leads to their dissemination in liver parenchyma, which precludes direct in vivo monitoring of grafted islets and makes it impossible to retrieve them for analysis (Figure 2C). To facilitate the identification of the mechanisms underlying islet graft resistance to DSA-mediated rejection in vivo, we relied on an alternative, well-validated model of islet grafting $(19,20)$, where purified pancreatic islets are placed under the capsule of the recipient's left kidney (hereafter referred to as the subcapsular model, Figure 3A). Of note in this model, left nephrectomy (i.e., islet graft removal) at the end of the follow-up period offers the opportunity to confirm the function of the graft and to rule out that glycemia control is due to neogenesis of insulin-producing $\beta$ cells in the native pancreas. Graft morphology analysis, performed 50 days after the procedure, showed large insulin-producing endocrine aggregates connected to cortical kidney vascularization (Figure 3B).

The subcapsular model recapitulated all the observations made when pancreatic islets were injected into the portal vein. Briefly, WT C57BL/6 mice tolerated syngeneic islet grafts (Figure $3 \mathrm{C})$. CBA islets were rejected by WT C57BL/6 recipients (syngeneic vs. allogeneic islet survival, $P<0.0001$, log-rank test; Figure $3 \mathrm{C})$ that generated DSA during the rejection process (Figure 3D). Finally, C57BL/6 RAG2 KO recipients did not reject CBA allogeneic islets (Figure $3 \mathrm{E}$ ), even when they were infused twice weekly with DSA-containing immune sera (Figure 3E).

The use of immune sera represented another limitation for evaluating the mechanisms underlying the resistance of grafted islets to humoral rejection. Indeed, the heterogeneity of the humoral responses generated by the recipient mice (Figure 2E and Figure 3D) made the standardization of the DSA transfer experiments more complex. Although we made substantial efforts to ensure that each recipient received a similar amount of DSA (Figure 2E and Figure 3D), it was difficult to control the stability of the "quality" (i.e., the spectrum of the heavy chain isotypes and their ability to activate the complement) of the different batches of pooled immune sera. Immune sera also limited the possibility of tracking DSA in vivo. The use of a commercially available purified mouse anti- $\mathrm{H}-2^{\mathrm{k}} \mathrm{mAb}$ permitted us to overcome these technical hurdles. The HB13 mAb, a murine IgG2a directed against $\mathrm{H}-2 \mathrm{~K}^{\mathrm{k}}$ and $\mathrm{D}^{\mathrm{k}}$ major histocompatibility complex class I molecules, was chosen for its ability to promote $\mathrm{Ab}$-mediated rejection lesions in $\mathrm{H}-2^{\mathrm{k}}$ heart transplants (21). In vitro, HB13 was comparable to pooled immune sera from WT C57BL/6 islet recipients in its ability to trigger complement-dependent lymphocytotoxicity (Figure 3F). However, since HB13 was slightly less efficient than pooled immune sera in inducing allogeneic islet cell destruction in vitro (Figure 3G), we set the amount of HB13 transferred i.v. to obtain a peak - a log higher than the median value of the DSA concentration observed in the WT C57BL/6 islet recipients (Figure $3 \mathrm{H}$ ). Infusions were performed i.v. every 72 hours to ensure 
A

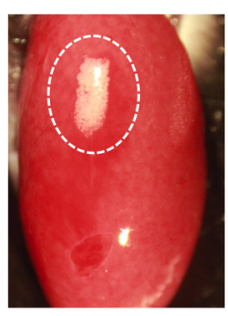

B

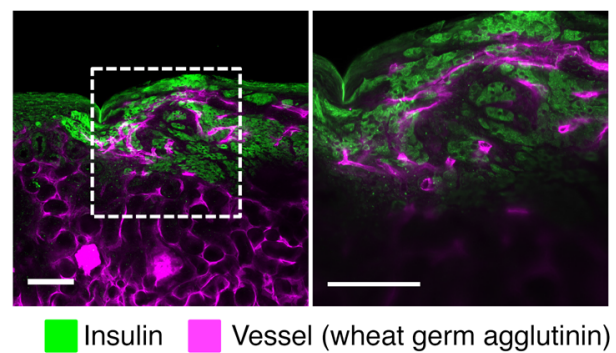

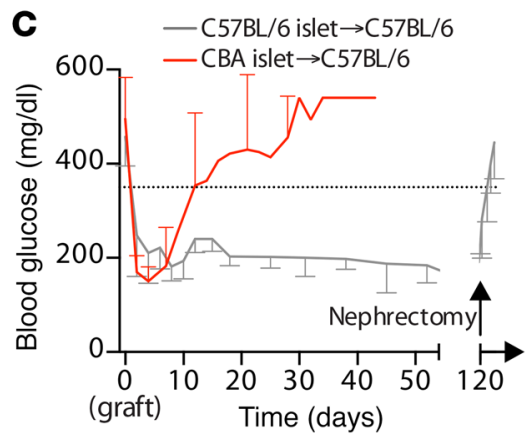

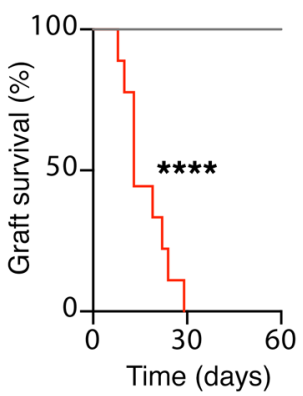

D

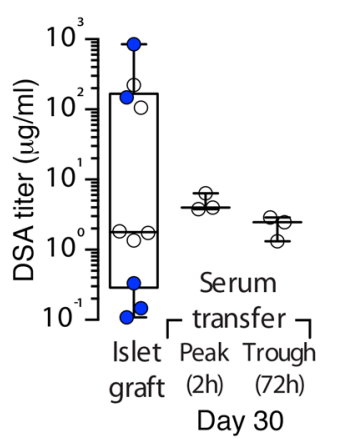

E

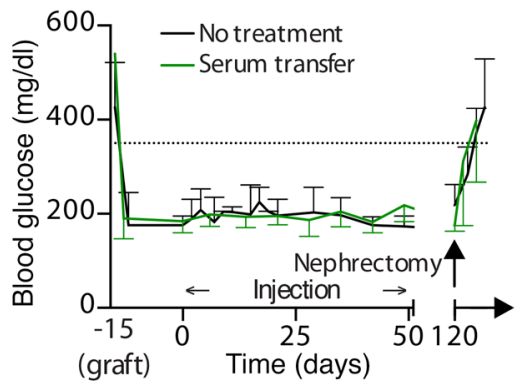

$\mathbf{F}$

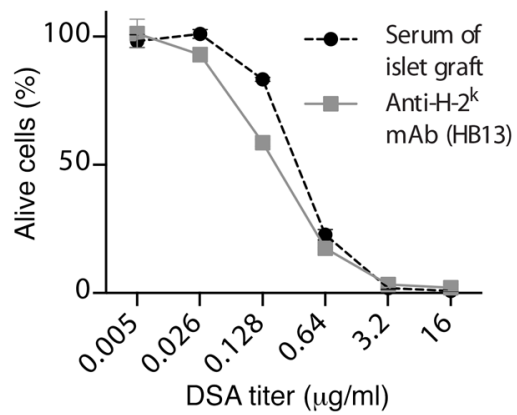

G

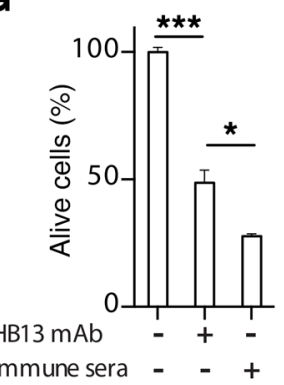

H

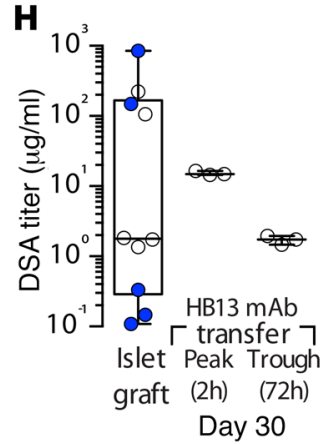

I

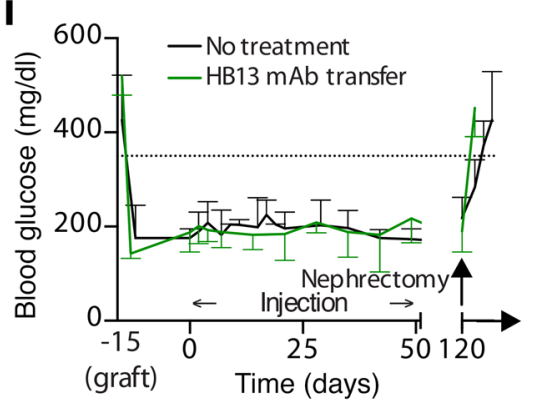

Figure 3. Optimization of experimental model. (A) Postoperative view showing pancreatic islets (white circle) grafted under left kidney capsule of recipients. (B) Left: representative finding of immunofluorescence analyses performed 50 days after subcapsular grafting of syngeneic islets. Right: magnification of the same. Scale bars: $100 \mu \mathrm{m}$. (C) Left: blood glucose levels were measured twice weekly in WT C57BL/6 recipients. Evolution of glycemia (mean \pm SD) is shown for C57BL/6 (syngeneic, gray; $n=6$ ) and CBA (allogeneic, red; $n=9$ ) subcapsular islet grafts. Islet graft loss was defined by fasting glycemia of more than $350 \mathrm{mg} / \mathrm{dl}$ (dashed line). Right: survival curves were compared. ${ }^{* * *} P<0.0001$, log-rank test. (D) Flow cytometry cross-match technique was used to quantify circulating DSA generated by WT C57BL/6 recipients in response to CBA subcapsular islet graft. Individual values are shown for 2 independent experiments (white and blue symbols). Peak and trough levels of circulating DSA were monitored 30 days after starting passive i.v. transfer of immune serum. (E) Evolution of glycemia (mean $\pm \mathrm{SD}$ ) is shown for C57BL/6 RAG2 KO mice grafted under the kidney capsule with CBA pancreatic islets and transferred (green; $n=3$ ) or not (black; $n=4$ ) with DSA. (F and $\mathbf{G})$ Cytotoxic potentials of immune serum and anti-H-2k $\mathrm{mAb}(\mathrm{clone} H B 13)$ were compared in vitro using complement-dependent cytotoxic assay (mean \pm SD) on CBA splenocytes (F) and CBA pancreatic islet cell suspension (G). ${ }^{*} P<0.05$; ${ }^{* * *} P<0.001$, 1-way ANOVA. (H) Peak and trough levels of circulating DSA were monitored 30 days after starting passive i.v. transfer of HB13. (I) Blood glucose levels were measured twice weekly in C57BL/6 RAG2 KO mice grafted under kidney capsule with CBA pancreatic islets. Evolution of glycemia (mean \pm $\mathrm{SD}$ ) is shown for recipients transferred (green; $n=4$ ) or not (black; $n=4$ ) with HB13.

that the titer of HB13 stayed above the median value of the DSA concentration observed in WT C57BL/6 islet recipients (Figure 3H). The i.v. infusions of HB13, like the passive transfer of immune sera, did not show any impact on CBA islet graft survival in the C57BL/6 RAG2 KO recipients (Figure 3I).

DSAs do not induce microvascular lesions in pancreatic islet graft in vivo. We next decided to compare the tissue damage triggered by DSA in grafted tissues versus transplanted organs. C57BL/6 RAG2 KO mice were used as recipients of either subcapsular CBA pancreatic islets or a CBA heart transplant. HB13 or PBS was infused i.v. twice weekly into recipient mice for 30 days and grafts/transplants were harvested for histological analysis. As expected, the PBS did not induce significant histological changes to the heart transplants and islet grafts. As previously reported $(21,22)$, the heart transplants exposed to DSA showed leukocyte margination in dilated capillaries (Figure 4A), which is typical of humoral rejection (23, 24). In contrast, the pancreatic islet grafts exposed to DSA in vivo were devoid of such microvascular lesions (Figure 4A). Immunohistochemistry confirmed that the grafted islets, in contrast with the heart transplants and despite the fact that they had similar 
A
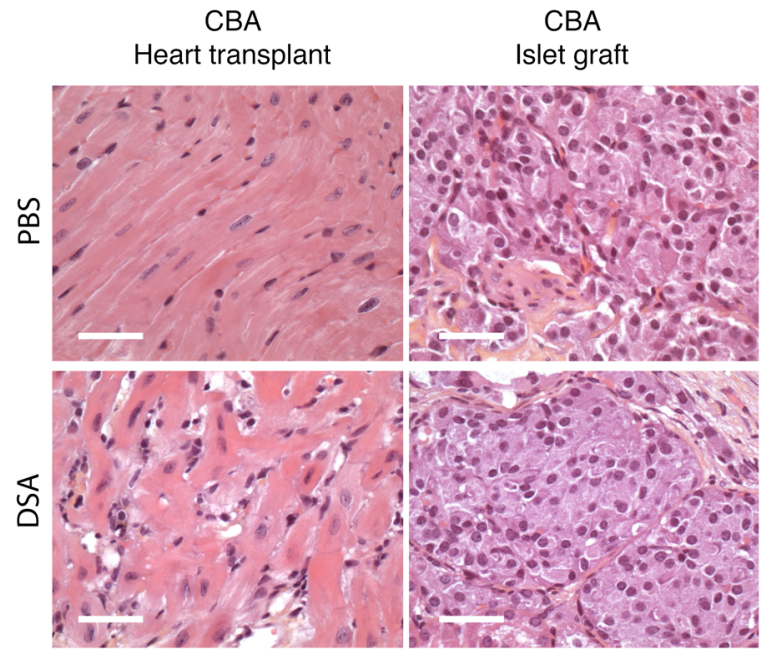

C
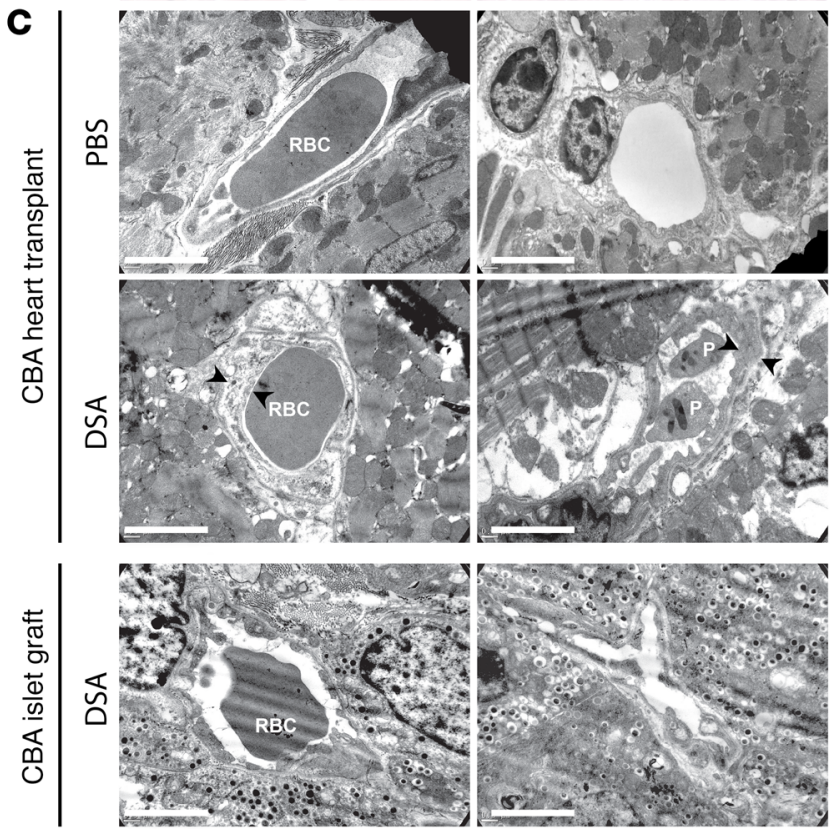

B
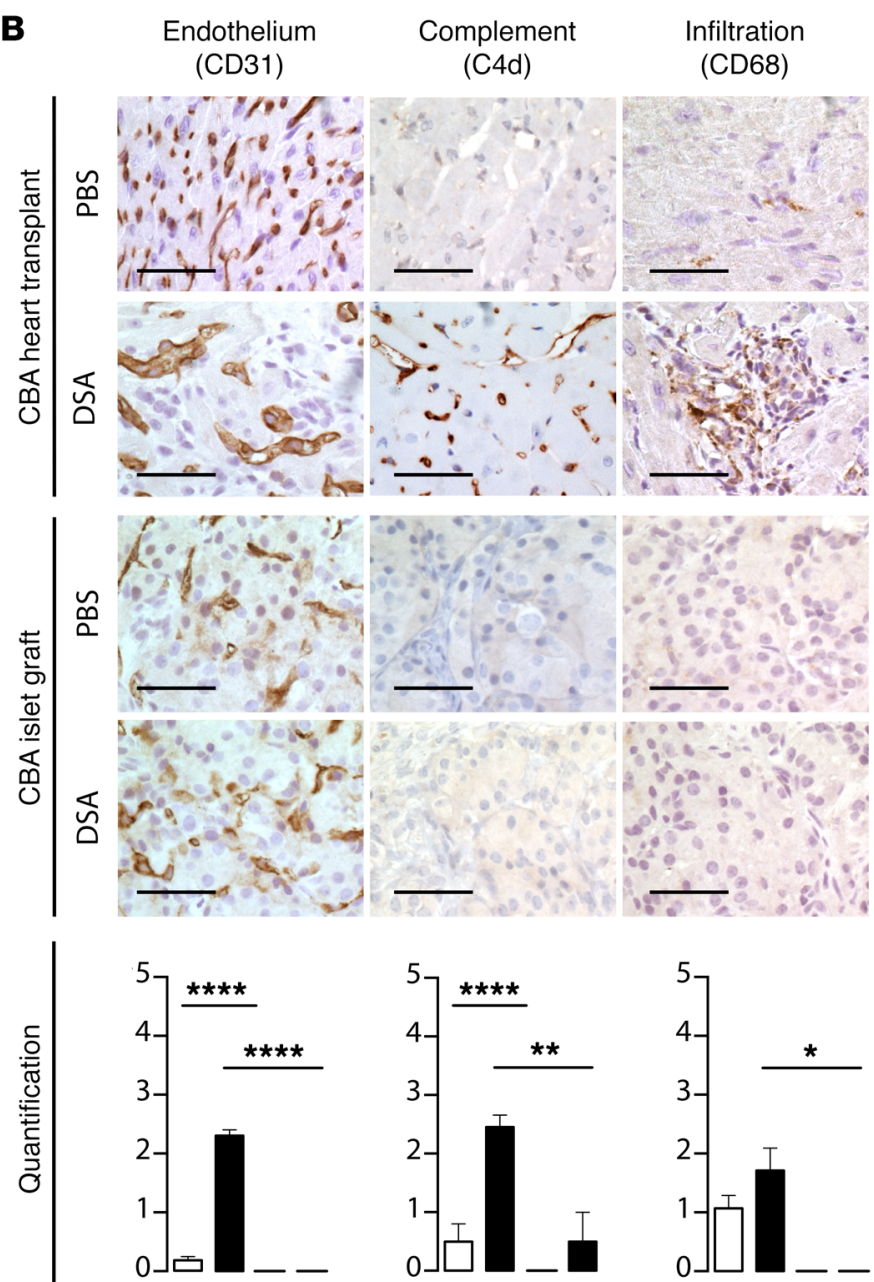
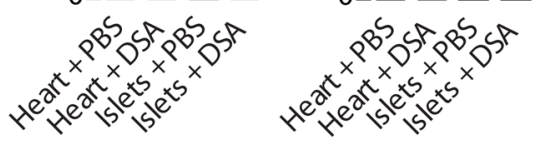

Figure 4. Histological evaluation of DSA-mediated lesions. C57BL/6 RAC2 KO mice were used as recipients of either a CBA subcapsular islet graft or a CBA heart transplant. HB13 or PBS was infused i.v. twice weekly into recipient mice for 30 days, and grafts/transplants were harvested for histological analysis. (A) Representative findings of H\&E stain are shown for the 4 experimental groups: (i) Heart + PBS, $n=13$; (ii) Heart + DSA; (iii) Islet + PBS, $n=11$; and (iv) Islet + DSA, $n=11$. Scale bars: $50 \mu \mathrm{m}$. (B) Immunohistochemistry was performed to evaluate the morphology of microvasculature (CD31), classical complement pathway activation (C4d), and macrophage infiltration (CD68). Representative findings are shown for the 4 experimental groups. Scale bars: $50 \mu \mathrm{m}$. A trained pathologist graded intensity of each elementary lesion on a semiquantitative scale (score 1-5). Mean \pm SD of the 4 experimental groups. ${ }^{*} P<$ 0.05 ; ${ }^{* *} P<0.01 ;{ }^{* * *} P<0.0001,2$-way ANOVA. (C) Transmission electron microscopy was used to assess the ultrastructural integrity of endothelial cells of CBA heart transplants (upper and middle rows) and CBA islet grafts (lower row) 30 days after the beginning of PBS (upper row) or HB13 transfer (middle and lower rows). Black arrowheads indicate swollen endothelial cells. P, adhesion of platelets. Scale bars: $3 \mu \mathrm{m}$.

microvessel density, did not develop endothelial turgidity, complement activation, or leukocyte infiltration upon DSA exposure (Figure 4B). In transmission electron microscopy, the microvasculature of heart transplants from recipients transferred with DSA showed distinctive features of Ab-mediated rejection, including swollen endothelial cells and platelet adhesion (Figure 4C). In contrast, endothelial cells of islet grafts from recipients transferred with DSA exhibited a normal ultrastructure, similar to that observed in the heart transplant of recipients injected with PBS (Figure 4C). Endocrine cells of islet grafts from recipients transferred with DSA also exhibited a normal ultrastructure (Supplemental Figure 4).
Endothelial chimerism protects grafted islets from DSA-induced vascular lesions in vivo. In contrast with transplantation, where perfusion is immediately reestablished by surgical reconnection of the arterial and venous vessels of the organ to the recipient's circulation, the restoration of blood flow to grafted islets involves angiogenesis (25-29). We postulated that this difference might explain the resistance of islet graft vasculature to the deleterious impact of DSA.

Syngeneic $\left(\mathrm{C} 57 \mathrm{BL} / 6, \mathrm{H}-2^{\mathrm{b}}\right)$ or allogeneic $\left(\mathrm{CBA}, \mathrm{H}-2^{\mathrm{k}}\right)$ hearts were transplanted into C57BL/6 RAG2 KO recipients. Four weeks after transplantation, the heart transplants were harvested and, following enzymatic digestion, the origin of the hematopoietic 
A
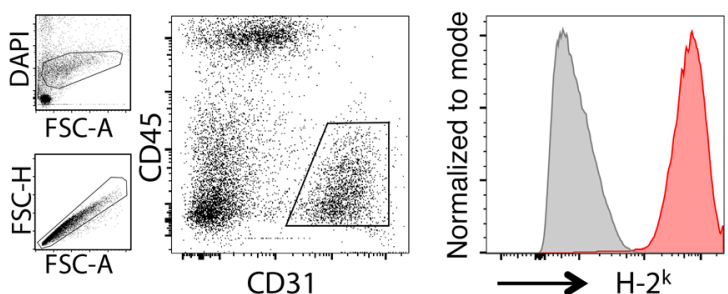

C57BL/6 heart transplant $\left(\mathrm{H}-2^{\mathrm{b}}\right)$

CBA heart transplant $\left(\mathrm{H}-2^{\mathrm{k}}\right)$

C

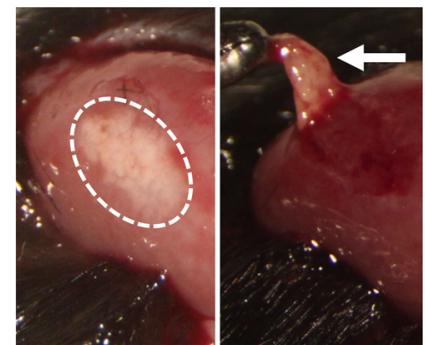

D
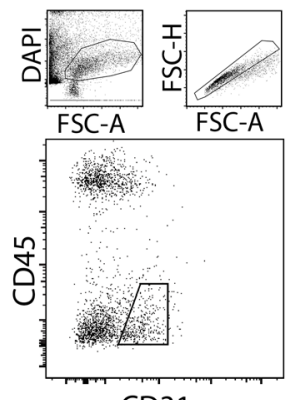

CD31
B

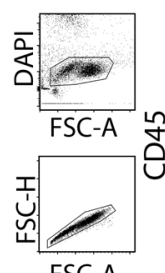

FSC-A

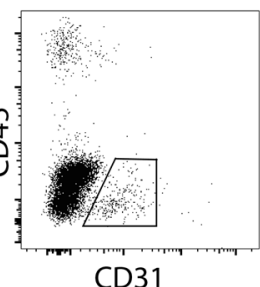

(Fresh)

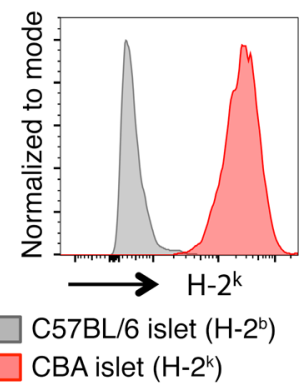

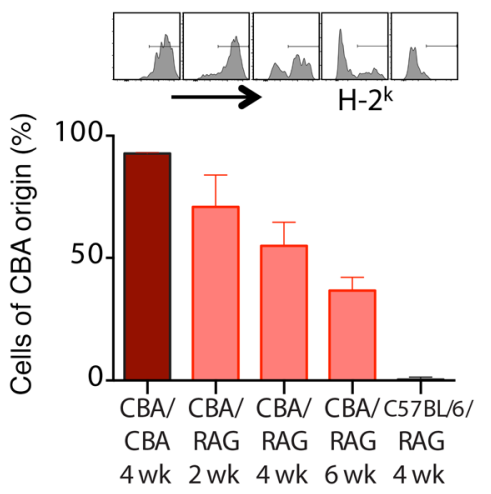

E

C57BL/6 islet graft $\left(\mathrm{H}-2^{\mathrm{b}}\right)$ CBA islet graft $\left(\mathrm{H}-2^{k}\right)$
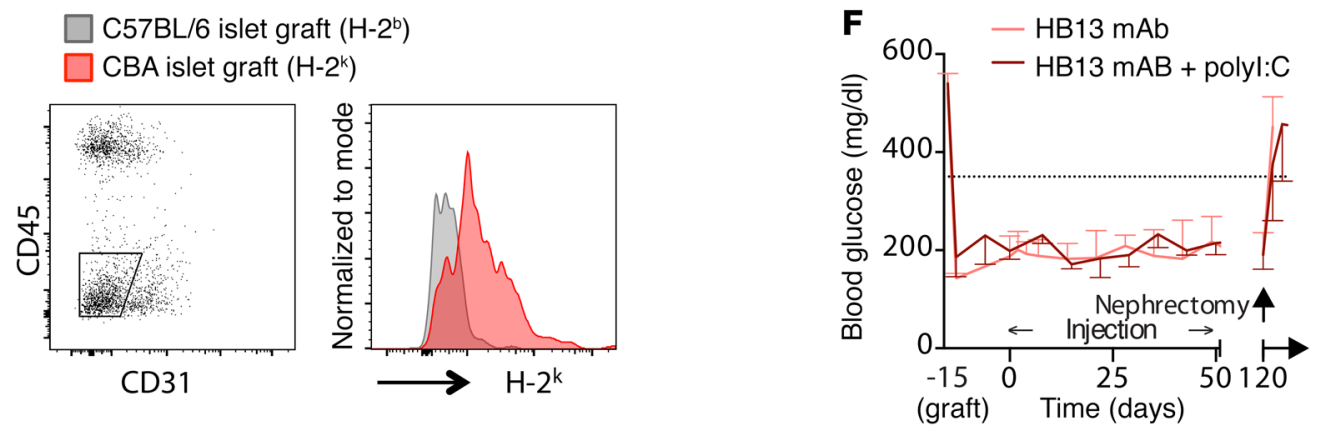

Figure 5. Endothelial chimerism in grafted islets. Each panel shows representative findings of 2 independent experiments. (A) C57BL/6 (gray) or CBA (red) heart was transplanted into C57BL/6 RAC2 KO recipients. Four weeks after transplantation, single-cell suspension was prepared by enzymatic digestion of the transplant and $\mathrm{H}-2^{\mathrm{k}}$ expression of $\mathrm{CD} 45^{-} \mathrm{CD} 31^{+}$endothelial cells were assessed by flow cytometry. (B) The same approach was used to analyze $\mathrm{H}-2^{\mathrm{k}}$ expression of endothelial cells of freshly isolated C57BL/6 (gray) or CBA (red) islets. (C) Subcapsular implantation made the retrieval of grafted islets possible: operative views of islet graft before (left, white dashed circle) and during (right, white arrow) microdissection. (D and E) C57BL/6 islets were transplanted to C57BL/6 RAG2 KO recipients (gray), and CBA islets were grafted either to CBA (dark red) or C57BL/6 RAG2 KO recipients (light red). (D) Grafted islets were microdissected at indicated time points, and the proportion of endothelial cells of CBA origin (i.e., $\mathrm{H}-2^{\mathrm{k}}$ positive) was assessed by flow cytometry. (E) The proportion of endocrine cells (CD45-CD31-) of CBA origin ( $\mathrm{H}-2^{\mathrm{k}}$ positive) was assessed in C57BL/6 (gray) and CBA (red) islets 6 weeks after grafting in C57BL/6 RAG2 KO recipient. (F) Blood glucose levels were measured twice weekly in C57BL/6 RAG2 KO mice grafted under the kidney capsule with CBA pancreatic islets. Evolution of glycemia (mean $\pm S D)$ is shown for recipients transferred with DSA alone (HB13 mAb, pink; $n=4)$ or in association with poly I:C (dark red; $n=3$ ). Nephrectomy was performed at 120 days to confirm grafted islet function.

$\left(\mathrm{CD}_{4} 5^{+} \mathrm{CD} 31^{-}\right)$, stromal $\left(\mathrm{CD}^{-} 5^{-} \mathrm{CD} 31^{-}\right)$, and endothelial cells $\left(\mathrm{CD} 45^{-} \mathrm{CD} 31^{+}\right)$was assessed by $\mathrm{H}-2^{\mathrm{k}}$ expression using flow cytometry (Figure 5A). As expected, the endothelial cells of the heart transplants were all of donor origin (Figure 5A). The same conclusion was reached when the same approach was applied to freshly isolated islets (Figure 5B).

Using the subcapsular model in the C57BL/6 RAG2 KO recipients, we were able to microdissect grafted islets, which allowed us to perform the analysis at various time points (Figure 5C). Even in the absence of rejection in these immunocompromised recipients, a progressive replacement of donor endothelial cells by the endothelial cells of recipient origin was observed within the graft- ed islets (Figure 5D). Donor endothelial cells indeed represented less than one-third of the intraislet endothelial cells 6 weeks after grafting. Since recipient endothelial cells do not express donorspecific allogeneic targets, the establishment of this endothelial chimerism explains the lack of microvascular lesions in pancreatic islet grafts exposed to DSA.

Endothelial cells of pancreatic islets are not directly responsible for graft function (i.e., the maintenance of a recipient's glycemic balance), which depends on the production of insulin by $\mathrm{CD} 45^{-} \mathrm{CD} 31^{-}$endocrine cells. In contrast with the endothelial cells, the endocrine cells of the CBA islet grafts expressed $\mathrm{H}-2^{\mathrm{k}} 6$ weeks after grafting in the C57BL/6 RAG2 KO recipients (Figure 5E), 
A
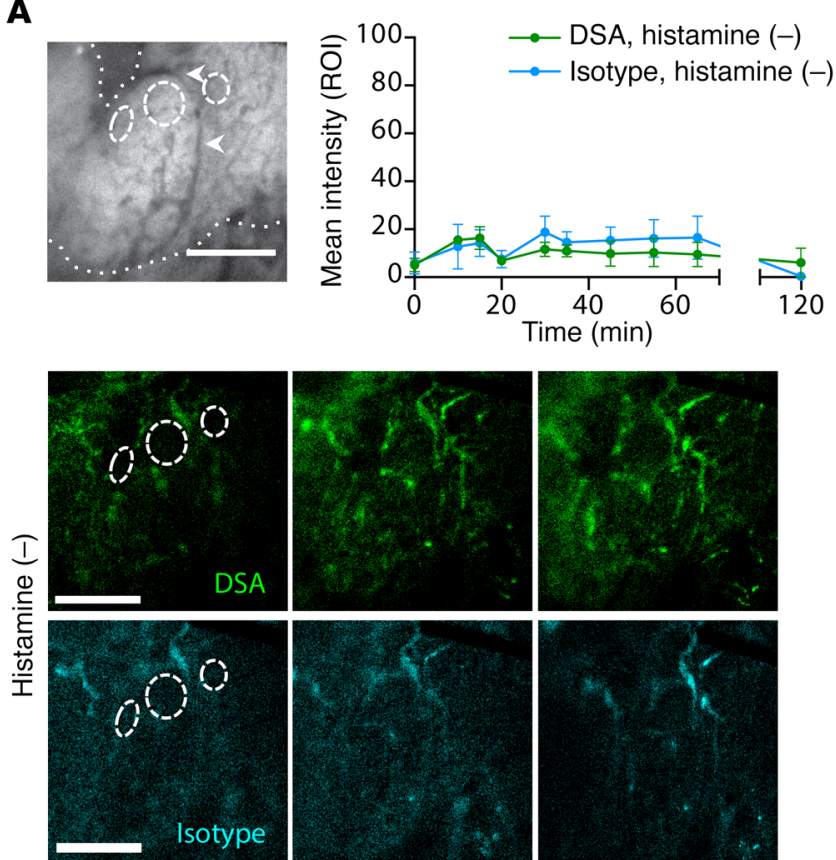

$10 \mathrm{~min}$

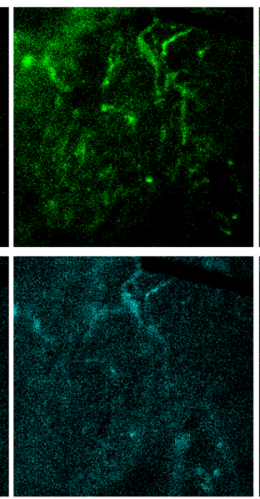

$30 \mathrm{~min}$

C

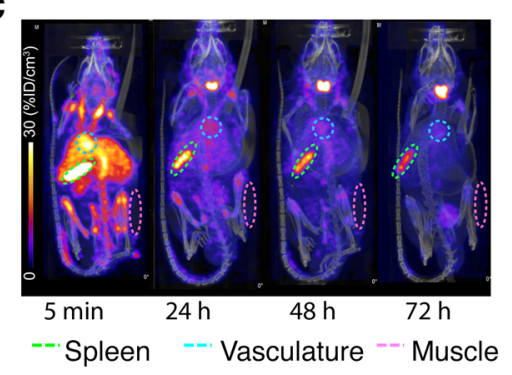

$60 \mathrm{~min}$
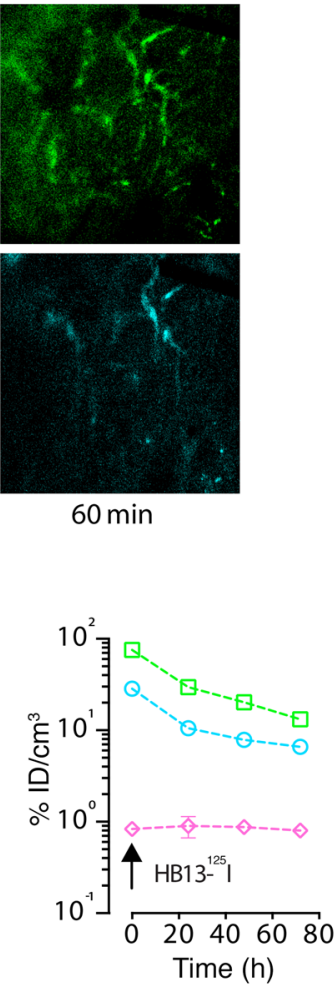

B
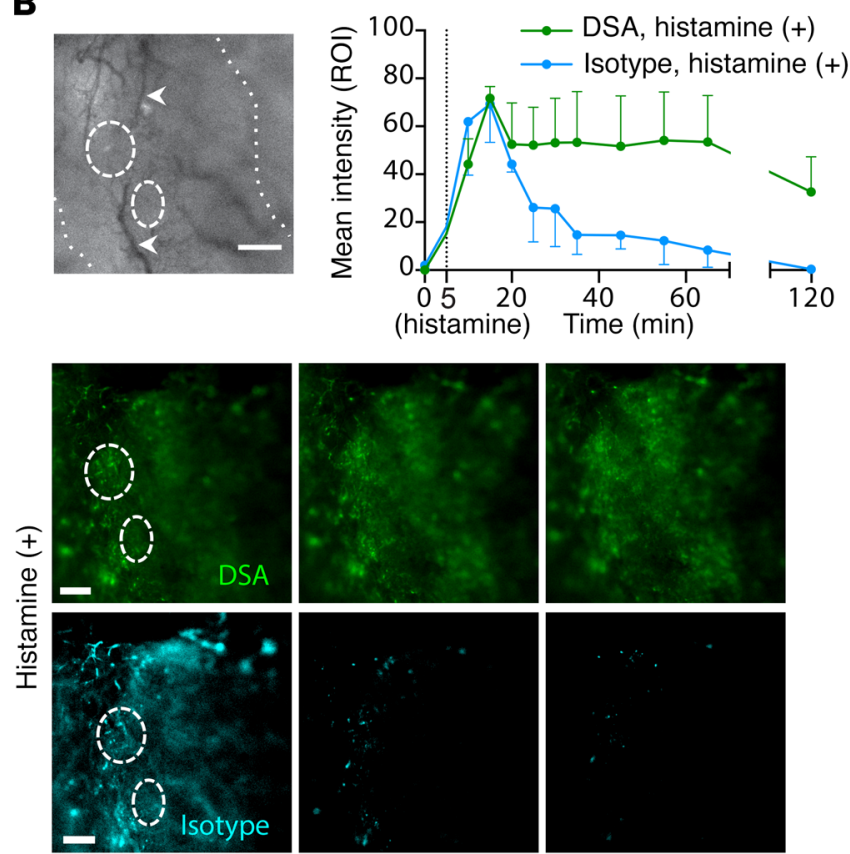

$10 \mathrm{~min}$
D

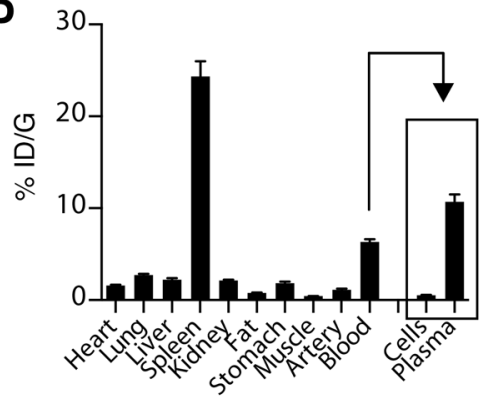

E

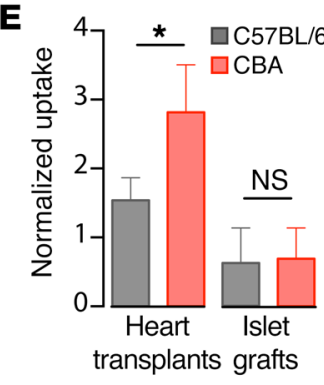

Figure 6. Vascular sequestration of DSA. (A) Fluorescently labeled DSA (HB13, green; $n=3$ ) and IgG2A isotype control (cyan; $n=3$ ) were infused simultaneously i.v. into C57BL/6 RAC2 KO mice previously grafted with CBA islets. Time-lapse intravital microscopy was used to monitor the intensity of fluorescence in several ROI. Upper left: representative bright field image showing islet graft outer limit (white dotted line) and ROI localization (white dashed circles), which were positioned outside islet graft vasculature (white arrowheads). Upper right: MFI in ROI was recorded from time of mAb injection $($ mean \pm SD). Lower rows: representative images showing vascular sequestration of DSA (upper row) and isotype control (lower row). Scale bars: $150 \mu \mathrm{m}$. (B) The same experiment was conducted as in A, except that histamine was locally applied on islet graft 5 minutes after beginning of recording. Groups are DSA (HB13, green; $n=3$ ) and IgG2A isotype control (cyan; $n=3$ ). Scale bars: $150 \mu \mathrm{m}$. (C) Biodistribution of i.v.-transferred iodinated HB13 (HB13-125I) was kinetically assessed in C57BL/6 RAC2 KO mice $(n=4)$ over 72 hours using SPECT/CT imaging. Left: representative images of SPECT analyses taken 5 minutes and 24,48 , and 72 hours after injection. Right: evolution of intensity of radioactive signals remaining in circulation (spleen, green; blood, blue) or extravagating in control tissue (muscle, purple) over time. (D) Quantification of radioactive signal in various tissues of C57BL/6 RAC2KO mice ( $n=4)$ measured 72 hours after i.v. injection of HB13-125. (E) Quantification of radioactive signal (mean \pm SD) measured in graft 72 hours after i.v. injection of HB13-125I. C57BL/6 RAG2KO recipients were transplanted with syngeneic (C57BL/6, $\mathrm{H}-2^{\mathrm{b}}$; gray; $\left.n=2\right)$ or allogeneic (CBA, $\mathrm{H}-2^{\mathrm{k}} ;$ red; $\left.n=3\right)$ hearts or grafted with syngeneic (C57BL/6, $\mathrm{H}-2^{\mathrm{b}}$; gray; $\left.n=2\right)$ or allogeneic (CBA, $\mathrm{H}-2^{\mathrm{k}}$; red; $n=3$ ) islets. ${ }^{*} P<0.05$, 1-way ANOVA.

demonstrating that they were of donor origin. While the expression of allogeneic targets should make endocrine cells sensitive to DSAmediated destruction, the lack of impact of DSA transfers on islet graft function (Figure $2 \mathrm{~F}$ and Figure $3 \mathrm{E}$ ) instead demonstrates that endocrine cells somehow show resistance to humoral rejection.

Increasing the expression of allogeneic targets on endocrine cells does not break islet graft resistance to humoral rejection in vivo. A first possible explanation for the lack of sensitivity of the graft endocrine cells to DSA was that these cells expressed significantly less allogeneic molecular targets than the endothelial cells (MFI of $\mathrm{H}-2^{\mathrm{k}}$ staining of endocrine vs. endothelial cells: $2762 \pm 2573$ vs. $59215 \pm 43506, P=0.012, t$ test). In vitro exposure of dissociated islet cells to $0.5 \mathrm{ng} / \mathrm{ml} \mathrm{IFN}-\gamma$ boosted the level of expression of MHC molecules (MFI of $\mathrm{H}-2^{\mathrm{k}}$ staining of endocrine cells before vs. after IFN- $\gamma: 5271 \pm 21932$ vs. $53535 \pm$ $53515, P<0.0001, t$ test; Supplemental Figure 5A). As expected, increasing the expression of allogeneic molecular targets on the dissociated islet cells made them more susceptible to DSAmediated destruction in vitro (percentage of live cells without vs. with IFN- $\gamma$ : $48.7 \pm 4.9$ vs. $5.7 \pm 0.4, P<0.05$, 1-way ANOVA; 
A

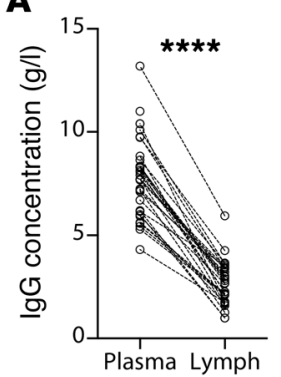

B

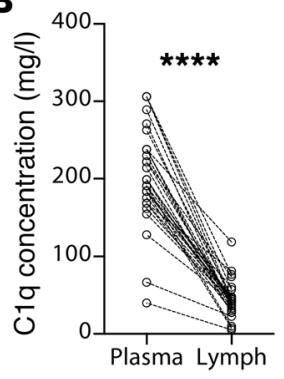

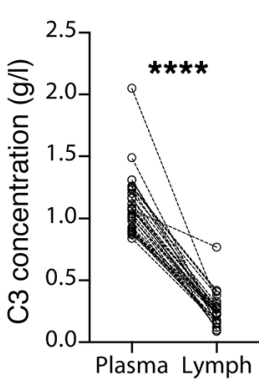

Figure 7. Vascular sequestration of IgG and complement components in transplanted patients. (A) Comparison of IgG content of paired plasma and lymph samples. ${ }^{* * *} P<0.0001$, paired $t$ test. (B) Comparison of the content in complement fractions C1q (left) and C3 (right) of paired plasma and lymph samples. ${ }^{* * *} P<0.0001$, paired $t$ test.

Supplemental Figure 5B). Similar findings were made when the experiments were conducted specifically with an insulin-producing $\beta$ cell line (Supplemental Figure 5, C and D).

To determine whether increasing the expression of allogeneic molecular targets on endocrine cells would be sufficient to break islet graft resistance to DSA in vivo, we relied on previously published works, which had reported that administration of polyinosinic:polycytidylic acid (poly I:C) to mice increased the IFN- $\gamma$ serum level up to a concentration of $1 \mathrm{ng} / \mathrm{ml}(30)$, similar to what we used for in vitro experiments. While i.p. administration of $100 \mu \mathrm{g}$ poly I:C reliably boosted MHC-I expression on pancreatic endocrine cells in vivo (MFI of $\mathrm{H}-2^{\mathrm{k}}$ staining without vs. with poly I:C: $2633 \pm 15306$ vs. $55077 \pm 54026, P<0.0001, t$ test; Supplemental Figure $5 \mathrm{E}$ ), coinjection of poly I:C with DSA did not increase the sensitivity of CBA pancreatic islet grafts to humoral rejection (Figure $5 \mathrm{~F}$ ). These results indicate that the level of expression of allogeneic molecular targets is not a key parameter in explaining islet graft resistance to DSA-mediated rejection in vivo.

Vascular sequestration of DSA protects islet grafts from humoral rejection in vivo. The impossibility of circulating DSA to reach the allogeneic targets expressed by endocrine cells could be another explanation for in vivo resistance of islet grafts to humoral rejection. Immunoglobulins are indeed massive polar proteins that have limited ability to diffuse outside the vascular bed. In line with this theory was the observation that DSA-mediated lesions in transfer experiments were exclusively concentrated in the vasculature of heart transplants layered by endothelial cells bearing allogeneic molecular targets (Figure 4, A and B).

To test this hypothesis, HB13 and an IgG2a $\kappa$ isotype control $\mathrm{mAb}$ were conjugated with small fluorescent tags that allowed in vivo tracking of labeled immunoglobulins (Supplemental Figure $6, \mathrm{~A}$ and $\mathrm{B})$. In vivo video-microscopy of mesenteric vasculature of C57BL/6 mice revealed that, following i.v. injection of DyLight 633-labeled isotype control mAbs, fluorescent mAbs were only detected within blood vessels (Supplemental Figure 6C and Supplemental Video 1). Only a local application of histamine, an organic nitrogenous mediator known to increase vascular permeability (31), resulted in progressive diffusion of the fluorescent $\mathrm{mAb}$ in the surrounding interstitial tissue (Supplemental Figure 6C and Supplemental Video 1).

In vivo video-microscopy was then used to monitor the distribution of fluorescence within the grafted pancreatic islets. DyLight 488-conjugated HB13 and DyLight 633-conjugated isotype control mAbs were coinjected i.v. to the C57BL/6 RAG2 KO recipients. Under baseline conditions, both fluorescent mAbs were only detected in the blood vasculature of the grafted islets (Figure 6A). Application of histamine solution on the grafted islets induced the diffusion of both fluorescent mAbs within the interstitial tissue of the islet grafts (Figure 6B). While the DyLight 633 signal progressively faded as the isotype control mAb was washed away by lymph flow, the DyLight 488 fluorescence remained stable, demonstrating that the DSAs were able to bind to the allogeneic targets expressed by the endocrine islet cells following extravasation from the circulation and were hence retained in the graft (Figure 6B).

Although these findings supported the theory of a vascular sequestration of DSA, in vivo video-microscopy did not allow for an extension of the tracking of $\mathrm{mAb}$ beyond a few hours. To rule out the existence of a slow diffusion of the DSA within the grafted islets, we conducted a second set of experiments in which the DSA was labeled with a $\gamma$-radioactive isotope of iodine-125 (HB13$\left.{ }^{125} \mathrm{I}\right)$. HB13 ${ }^{-125}$ I was injected i.v. into C57BL/6 RAG2 KO mice and the radioactive signal was kinetically assessed over 72 hours using SPECT/CT imaging (Figure 6C). HB13-125I was only detected in the blood vasculature of the animals (Figure 6C). To further confirm the very limited ability of DSA to diffuse outside the vascular bed, we directly measured the radioactive signal emitted by various tissues of C57BL/6 RAG2 KO mice at 72 hours after i.v. injection of HB13-125I. In line with previous data, the HB13-125 I signal was almost entirely detected in the vascular system (i.e., spleen and blood; Figure 6D). Analyzing more precisely which fraction of the blood contained the radioactive signal, we observed that the HB13-125 I was in the plasma rather than in the cellular fraction, ruling out the possibility that the vascular sequestration of the DSA was the consequence of immunoglobulin binding to $\mathrm{Fc}$ receptors of peripheral blood mononuclear cells (Figure 6D). Finally, HB13-125I was injected i.v. into the C57BL/6 RAG2 KO recipient of either an islet graft or a heart transplant. While the CBA heart displayed an increased radioactive signal as compared with the control syngeneic heart (Figure 6E), the islet to blood ratios were similar for the CBA and C57BL/6 islet grafts, thereby demonstrating that allogeneic stromal cells from the graft are inaccessible targets for DSA.

Vascular sequestration of DSA and complement components in transplanted patients. In an attempt to validate the vascular sequestration of DSA in the clinic, we compared the composition of 32 paired plasma and lymph samples from recently transplanted renal recipients. The lymph samples, obtained from surgical drainage, were used as surrogates for interstitial fluid. The lymph concentration of IgG (molecular weight: $150 \mathrm{kDa}$ ), was only one-third of the plasma concentration (plasma vs. lymph: $7.85 \pm 1.94$ vs. $2.67 \pm$ $1.06 \mathrm{~g} / 1, P<0.0001$, paired $t$ test; Figure 7A). Seven patients from the cohort were sensitized against allogeneic HLA molecules not expressed by their renal allograft, which offered the opportunity to specifically evaluate the diffusion of anti-HLA Abs in tissues. Of the 72 distinct anti-HLA Ab specificities detected in these 7 plasma samples (52 anti-HLA II and 20 anti-HLA I), 56 (78\%) were also present in the paired lymph samples, but at lower titers (Supplemental Figure 7A). Of the 16 anti-HLA specificities that were miss- 
A
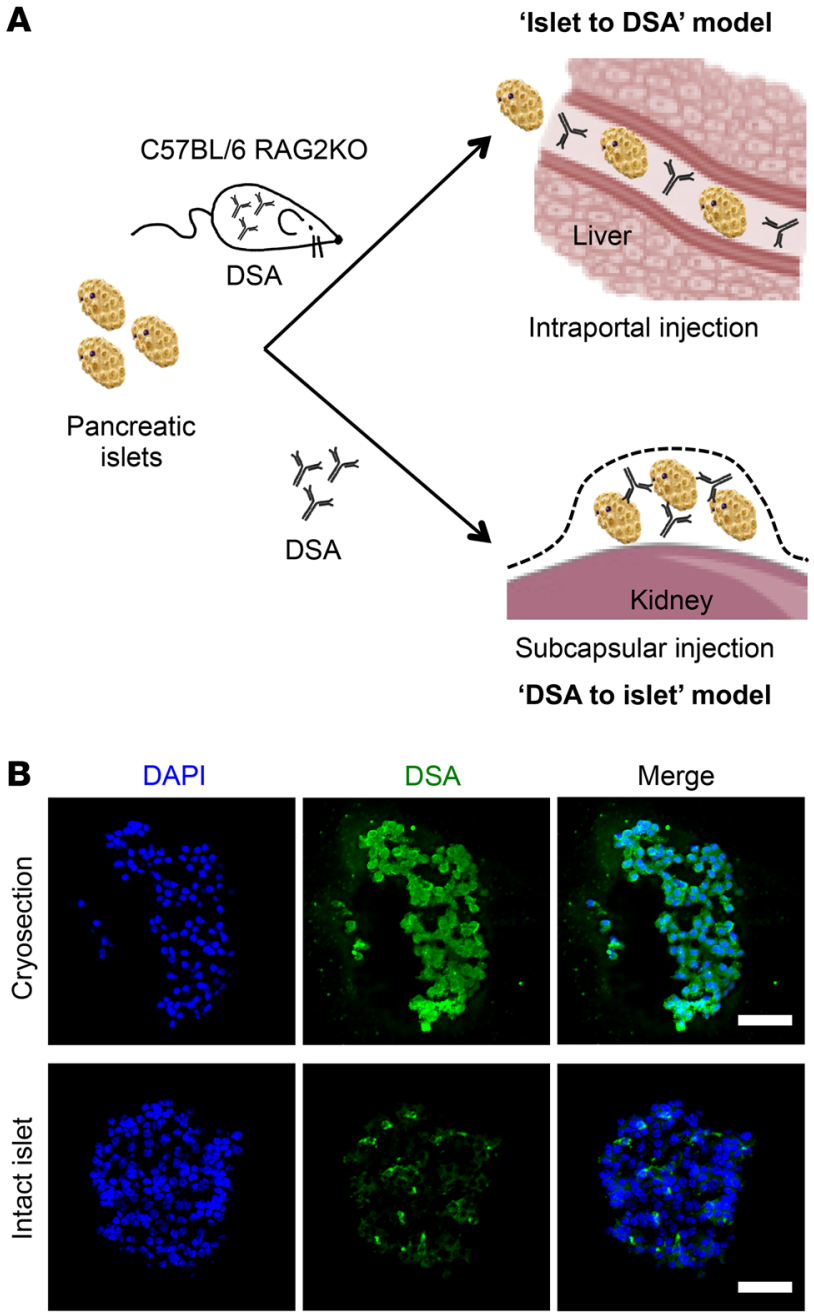

ing in the lymph, 9 (56\%) were directed against HLA I, and 7 (44\%) against HLA II. All 7 patients had at least 1 (range: 1 to 4 ) anti-HLA specificity missing in their lymph repertoire. One patient had antiHLA Abs only in the circulation. Comparison of anti-HLA Ab titers for the paired plasma and lymph samples showed a positive correlation in the linear regression model $(P<0.0001$; Supplemental Figure $7 \mathrm{~B}$ ), suggesting that vascular sequestration of anti-HLA Abs resulted from a passive barrier effect.

Several recent clinical studies have highlighted the importance of the classical complement cascade in the pathophysiology of DSA-mediated rejection (16-18). The binding of C1q to DSA fixed on alloantigens initiates the cascade, which results in the assembly of the classical pathway C3 convertase. The latter cleaves C3 into $\mathrm{C} 3 \mathrm{a}$, a potent proinflammatory mediator that causes leukocyte recruitment, and $\mathrm{C} 3 \mathrm{~b}$, leading to the formation of membrane attack complexes. Interestingly, C1q and C3 are both large proteins whose molecular weights (respectively, 400 and $186 \mathrm{kDa}$ ) exceed the molecular weight of IgG. Accordingly, these 2 activators of the classical complement cascade had even lower lymph/plasma concentration ratios than that of $\operatorname{IgG}$ (IgG vs. C3 vs. C1q, $0.35 \pm 0.11$ vs. $0.24 \pm 0.11$ vs. $0.22 \pm 0.10, P<0.0001,1$-way ANOVA; Figure 7B).

These findings indicate that large human proteins, including Abs and activators of the complement cascade, have limited abil-

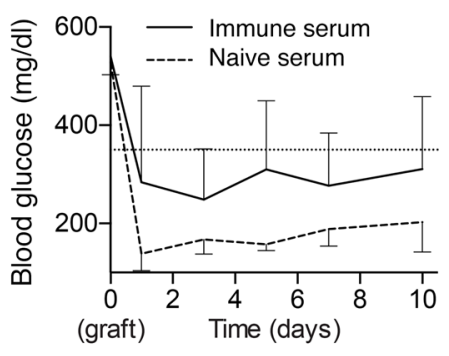

Figure 8. Direct contact between immune serum and allogeneic targets restores in vivo DSA toxicity. (A) CBA islets were grafted to C57BL/6 RAG2 KO recipients. Left panel: the 2 models used to establish a direct contact between immune serum and islet grafts are presented. Right panels: in the 2 models, blood glucose levels were measured twice weekly in islet graft recipients. Evolution of glycemia (mean \pm SD) is shown for mice transferred with naive (dashed line; $n=5$ ) or immune (solid line; $n=5$ ) serum. (B) The ability of DSA to diffuse through islets was tested by incubating in vitro HB13 with freshly isolated intact CBA pancreatic islets (lower row). Positive control (upper row) is a cryosection of CBA islets stained with HB13. Scale bars: 100 $\mu \mathrm{m}$. (C) Cytotoxic potential of the immune sera was assessed in vitro using complement-dependent cytotoxic assay on intact CBA islets or islet cell suspensions. ${ }^{* *} P<$ $0.01 ;{ }^{* * *} P<0.0001,1$-way ANOVA (mean \pm SD). ity to diffuse outside the vascular bed. Importantly, it shall be noted that vascular sequestration of large proteins is likely underestimated in our analyses because the lymph samples were obtained from surgical drainages that were contaminated with blood traces (as assessed by the presence of red blood cells, $123 \cdot 10^{3} \pm 203 \cdot 10^{3} / \mathrm{mm}^{3}$ ).

Direct contact of immune serum with allogeneic graft restores DSA toxicity in vivo. From the data presented above, we hypothesized that the resistance of allogeneic islets to humoral rejection could result from the synergistic combination of (i) the sequestration of DSA and complement activators within the circulation that restrains their deleterious impact on the allogeneic targets expressed by graft vasculature and (ii) the fact that islet graft vascularization developed mostly from the recipient.

We reasoned that if this theory were correct, establishing a direct contact between immune serum and allogeneic targets expressed by graft endocrine cells should restore DSA toxicity in vivo. To test this hypothesis, we used 2 complementary approaches (Figure 8A). In the first model (Figure 8A), CBA islets were injected into the portal vein of C57BL/6 RAG2 KO recipients that had previously been transferred with naive or immune serum. In the second model (Figure 8A), CBA islets were coinjected under the kidney capsule of C57BL/6 RAG2 KO recipients with naive or immune serum. In line with the hypoth- 
esis, islet graft function was drastically impaired in both models when immune (but not naive) serum was placed in direct physical contact with allogeneic islets (Figure 8A).

However, in both models, the allogeneic islets maintained some residual function (Figure 8A), suggesting that in addition to vascular sequestration, there might be an additional difficulty for DSA to diffuse through an intact islet structure. In line with this theory, (i) immunofluorescence analyses revealed that DSA bound less efficiently to intact islets than to cryosections (Figure $8 \mathrm{~B}$ ) and (ii) the cytotoxic impact of DSA in vitro was 2.5 times higher on islet cell suspensions than on intact islets (Figure 8C).

\section{Discussion}

Pancreatic islet grafting represents an attractive therapeutic alternative to pancreas transplantation for type I diabetic patients. Analyzing a cohort of 49 pancreatic islet graft recipients, we observed that, although these patients can generate Abs against donor-specific HLA molecules, DSA appearance did not correlate with shortened survival time of the islet grafts. This finding is in striking contradiction with the situation of solid organ transplantation $(9,10)$, including pancreas transplantation $(32,33)$, for which DSA-mediated rejection is unanimously recognized as the first cause of graft failure.

Using murine experimental models of pancreatic islet grafting, we demonstrated that the humoral response of recipients was functional, but we identified graft characteristics that synergize to protect allogeneic islets from DSA-mediated destruction. First, the graft endocrine cells constitutively expressed low levels of allogeneic targets (i.e., donor MHC molecules). This factor alone was, however, insufficient in solely explaining islet graft resistance to humoral rejection, since immune sera from the islet graft recipients efficiently destroyed the allogeneic islet cells in vitro and an increase of MHC expression on the islet cells failed to break the resistance of the islet grafts to the DSA in vivo. Instead, we concluded that the resistance of allogeneic islets to humoral rejection was explained by the impossibility of DSA and complement activators reaching allogeneic targets expressed by islet endocrine cells. These large proteins indeed have a poor ability to diffuse outside the circulation and through the islet structure, which explains why histological lesions of humoral rejection are restrained to the immediately accessible allogeneic targets expressed by the vasculature of transplanted organs. Graft, however, differs from transplant inasmuch as no vascular anastomosis is performed at the time of the surgical procedure. Reestablishment of islet blood flow, which is critical for the survival and function of grafted islets, occurs through sprouting of capillaries of recipient origin (25-27) that are not allogeneic. Although some donor endothelial cells persist and can be integrated within newly formed microvessels $(28,29)$, they are only a minor fraction of the graft endothelial cells. In our model, where islets were grafted to recipients devoid of an adaptive immune system, the donor origin cells represented approximately $30 \%$ of graft endothelium 6 weeks after the procedure (Figure 5D). It is likely that the presence of DSA in the recipient's circulation accelerates the elimination of donor endothelial cells, which could explain the total lack of microvascular lesions observed in Figure 4. Another possible explanation could be that healthy (i.e., recipient) endothelial cells, when numerically domi- nant, provide paracrine signals that protect the rest of the endothelium, including the few remaining donor endothelial cells.

What could be the consequences of our findings in the clinic? The inexorable decline of islet graft function over time currently represents a major challenge in the field. Several lines of evidence indicate that, as with solid organ transplantation, the recipient's alloimmune response is a major contributor to islet graft loss (8, 34). However, contrary to what occurs in transplanted organs, for which cellular rejection represents a marginal cause of graft loss (35), our data indicate that cellular rejection is both necessary and sufficient for the destruction of grafted islets. This conclusion is congruent with previous clinical observations (36) and carries important clinical implications. In solid organ transplantation, histological examination is the gold standard for the diagnosis of cellular rejection. In contrast, in clinical islet grafting, liver biopsies have not entered clinical routine for monitoring rejection. Indeed, only 5 to $10 \mathrm{~g}$ of purified pancreatic islets are injected into the portal vein of the recipient, leading to their dissemination within the $1.5 \mathrm{~kg}$ of liver parenchyma. Consequently, the chance for a percutaneous needle biopsy to sample an islet is estimated below 5/1,000. Although several techniques have been developed to monitor antiHLA reactivity of peripheral blood lymphocytes (37-39), these surrogate approaches all involve the realization of labor-intensive and complex in vitro assays, precluding their routine use. Hence, rather than the treatment, it is the diagnosis of cellular rejection that seems to be the main hurdle to the improvement of islet graft survival. To differentiate into DSA-producing plasma cells, B cells need to receive the help of follicular helper T cells. DSA appearance in the circulation, which can be easily monitored (40), therefore reflects an insufficient blockade of alloreactive $\mathrm{T}$ cells by immunosuppressive drugs. Hence, it is tempting to speculate that DSAs, despite the fact they are devoid of a direct pathogenic effect on grafted islets, might be valuable biomarkers for the diagnosis of $\mathrm{T}$ cell-mediated rejection. Whether DSA monitoring could guide the initiation of rejection treatment in islet recipients, however, remains to be tested because (i) the $\mathrm{T}$ cell subset providing help to $\mathrm{B}$ cells is not the one responsible for $\mathrm{T}$ cell-mediated rejection and the sensitivity to immunosuppressive drugs of these 2 subsets might be different and (ii) islet graft destruction in the clinic can also be mediated by the recurrence of type I diabetes, i.e., by autoreactive $\mathrm{T}$ cells, which are not involved in DSA generation. Interestingly, a recent clinical study has established that monitoring the fluctuations of DSA and autoantibody titers reliably identified islet graft recipients with a higher risk for rapid graft failure (14).

We believe that our study also has more general implications in immunology. Our data indeed indicate that the reduced concentrations of several key components of the humoral response in interstitial tissue make it resistant to Ab-mediated lesions. This theory fits with seminal clinical observations made in the field of solid organ transplantation in which humoral rejection was first named "vascular" rejection because most histologic damages were concentrated on the vasculature of the transplants (41). Physiologically, vascular sequestration of Abs could contribute to the maintenance of immune tolerance in the presence of autoreactive $B$ cell clones, which have repeatedly been observed in the humoral repertoire of healthy subjects (42). It is also tempting to speculate that the difficulty for the immune system of transferring 
a sufficient amount of $\mathrm{Ab}$ from the circulation to the site of tissue damage has been a driving evolutionary force for lymphoid neogenesis: the process by which ectopic "tertiary lymphoid organs" appear within chronically inflamed tissues (43), including rejected grafts (44), and serve as a site of local Ab generation (44-47). Vascular sequestration of immunoglobulins and complement activators could have important implications for cell therapies. A recent study reported that allogeneic-induced pluripotent stem cell-derived cardiomyocytes could regenerate nonhuman primate myocardium when directly injected in infarcted heart (48). Allogeneic cardiomyocytes survived for 12 weeks with no evidence of immune rejection in immunosuppressed monkeys. Interestingly, the authors showed that recipient primates formed endothelial cells of host origin within grafted cells, which could have participated in their resistance to humoral rejection. On the other hand, the very same process likely represents an important obstacle to the efficiency of biotherapies such as vaccines and mAbs $(49,50)$. An increase in the vascular permeability of the targeted tissue might represent an interesting approach for boosting the therapeutic efficiency of these strategies. Supporting this concept, a recent experimental study has demonstrated that mice that have developed Abs against herpes simplex virus type 2 after vaccination failed to survive a new challenge with the virus in the absence of $\mathrm{CD} 4^{+} \mathrm{T}$ cells. The authors demonstrated that $\mathrm{CD} 4^{+} \mathrm{T}$ cells were indeed necessary to enable Ab delivery to the infection sites by secreting IFN- $\gamma$ and enhancing microvascular permeability (51). Likewise, a synergy between allogeneic $\mathrm{T}$ cells and alloantibodies may be at work in the context of organ rejection.

\section{Methods}

Human study design. To determine the impact of DSA on islet graft survival, we retrospectively reviewed the medical files of all patients that received an islet graft between 2000 and 2016 within the GRAGIL Swiss-French multicenter network. Inclusion criteria were (i) successful islet grafting defined as insulin independence after the procedure for 2 years or more and (ii) availability of biological material for analysis of DSA response (HLA typing of donor and recipient, plus exhaustive annual sera samples). Forty-nine patients were identified (characteristics of the cohort are presented in Supplemental Table 1), among which 9 developed de novo DSA, all during the first year after grafting. Islet graft function was monitored longitudinally using the $\beta$ score, a validated composite score that integrates insulin requirements, C-peptide secretion, and glycemic control (15).

To compare the concentrations of different proteins in plasma and lymph, we prospectively enrolled 32 consecutive patients who underwent kidney transplantation at the Lyon University Hospital. Seven of these patients had preformed anti-HLA Abs that were not directed to the donor. Lymph was obtained when the surgical drain was withdrawn (postoperative days 3-7). On the same day, plasma was collected from routine blood examination.

Comparison of lymph and plasma. The concentrations of IgG and C3 were measured by immunonephelometry assay with BN ProSpec (Siemens) and C1q by the Radial Immunodiffusion Assays Kit for human complement functional assays (The Binding Site).

Detection and characterization of anti-HLA Abs. Centralized analyses were performed in a blinded fashion by a single trained immunobiologist (V. Dubois) at the French National Blood Service.
Sera and lymph samples were analyzed using Single Antigen Flow Beads (Lifecodes Single Antigen class I and class II; Immucor). The MFI was measured on a LABScan 100 (One Lambda).

Animals. WT C57BL/6 (H-2 $\left.{ }^{\mathrm{b}}, \mathrm{CD} 45.2\right), \mathrm{C} 57 \mathrm{BL} / 6$ Ly5.1 (H-2 ${ }^{\mathrm{b}}$, $\mathrm{CD} 45.1)$, and CBA $\left(\mathrm{H}-2^{\mathrm{k}}\right)$ mice aged 8 to 15 weeks were purchased from Charles River Laboratories. RAG2 KO mice on a C57BL/6 background were obtained from Cryopreservation Distribution Typage et Archivage Animal. All mice were maintained under EOPS (Exemption of Specific Pathogenic Organisms) conditions in our animal facility: Plateau de Biologie Expérimentale de la Souris (http://www.sfr-biosciences.fr/ plateformes/animal-sciences/AniRA-PBES; Lyon, France).

Experimental murine models. Heterotopic heart transplantations were performed as in the literature (52).

Diabetes was induced by a single i.p. injection of streptozotocin (170 $\mathrm{mg} / \mathrm{kg}$ ). Recipient mice were considered diabetic when fasting glycemia was measured as more than $350 \mathrm{mg} / \mathrm{dl}$. Blood glucose levels were monitored twice a week with a FreeStyle Optium glucometer (Abbott).

For pancreatic islet isolation, $2 \mathrm{mg}$ collagenase type XI (SigmaAldrich) dissolved in $2 \mathrm{ml}$ Hank's solution was perfused into the pancreas of the donor animal by cannulating the common bile duct. The pancreas was then removed and incubated at $37^{\circ} \mathrm{C}$ for 12 minutes. Islets were isolated by gradient density with Histopaque 1077.

After isolation, islet equivalents (IEQ) were either injected into the portal vein of the recipient (550 IEQ/recipient) or placed in the subcapsular space of the left kidney (250 IEQ/recipient).

Adoptive transfer of $T$ lymphocytes. A detailed protocol used for adoptive transfer of $\mathrm{T}$ cells is provided in Supplemental Methods.

Passive transfer of DSA. Transferred DSAs were either polyclonal (immune sera), collected after primary or memory responses, or monoclonal (clone HB13 directed against $\mathrm{H}-2 \mathrm{~K}^{\mathrm{k}}$ and $\mathrm{D}^{\mathrm{k}}$; BioXcell). Infusions of i.v. immune sera were started 15 days after the grafting procedure, when the vascularization of islet graft had just been reestablished.

A single batch of immune serum was used for a given experiment. Each batch was prepared by pooling the serum of 6 C57BL/6 mice sensitized against $\mathrm{H}-2^{\mathrm{k}}$. DSA titer was estimated for every batch using a custom flow cross-match assay and a standard curve derived from known concentrations of HB13 (Supplemental Figure 1).

DSAs were passively transferred to recipients by retroorbital injection until the end of the follow-up. The amount of DSA transferred and the frequency of infusions were set to ensure that the titer of circulating DSA remained stable and similar to the median value observed in WT recipients grafted with allogeneic islets.

Avidity of DSA collected after primary or memory alloimmune responses was estimated by measuring the stability of preformed antigen-Ab complexes in the presence of increasing concentrations of a chaotropic agent, as reported previously (53).

For further details, see Supplemental Methods and Supplemental Figure 3.

Flow cytometry. Detailed protocols used for flow cytometry analyses are provided in Supplemental Methods.

In vitro cytotoxicity assay. For the in vitro cytotoxicity assays, CBA splenocytes, intact CBA islets, CBA islet cell suspensions, or the $\mathrm{H}-2^{\mathrm{k}}$ restricted $\beta$ TC-tet cell line (54), a gift from Bernard Thorens (University of Lausanne, Lausanne, Switzerland), were incubated with immune serum or HB13 mAbs for 1 hour at $4^{\circ} \mathrm{C}$. When indicated, target cells were precultured 24 hours in $0.5 \mathrm{ng} / \mathrm{ml} \mathrm{IFN-} \gamma$ (Peprotech) to enhance MHC class I expression. 
After washing excess Abs, rabbit complement (Cedarlane) was added at $1 / 16$ dilution for 1 hour at $37^{\circ} \mathrm{C}$.

Dead cells were stained with DAPI, and the percentage of viable cells was evaluated by flow cytometry. Survival rate was normalized to the results observed when cells were incubated with complement alone.

Pathological analyses. Detailed protocols used for the pathological analyses are provided in Supplemental Methods.

Transmission electron microscopy of the left kidney (containing grafted islets) was performed as previously described (55).

Intravital microscopy. Mice were anesthetized by ketamine/xylazine injection. Abdominal midline incision was performed to pull the small intestine out, or a left flank incision was made to push the left kidney out. Mesenteric vessels of left kidney (containing grafted islets) were exposed under an upright fluorescence macroscope (MacroFluo, Leica Microsystems) equipped with a thermostatic plate and a $5 \times$ lens, which was connected to an sCMOS camera (Orca-Flash-4.0, Hamamatsu Photonics), and images were acquired by Metamorph and processed by Image (NIH). HB13 mAbs labeled with a DyLight 488 Fast Conjugation Kit (Abcam) and isotype control (mouse IgG2aא, BioLegend) labeled with a DyLight 633 Fast Conjugation Kit (Abcam) were infused simultaneously by retroorbital injection. When indicated, $2 \mu$ l of histamine $(100 \mathrm{nM})$ was applied onto the observed tissue.

Nuclear imaging. HB13- ${ }^{-125}$ I was prepared using a direct iodination procedure with Iodogen reagent (PerkinElmer) as an oxidant. Briefly, $37 \mathrm{MBq}$ of ${ }^{125} \mathrm{I}$ was added to $20 \mu \mathrm{g}$ of HB13 in phosphate buffer (50 $\mathrm{mM}$ ) and the mixture was then transferred into a glass tube precoated with Iodogen. The reaction was allowed to proceed at room temperature for 15 minutes. Radioiodinated HB13 was then purified through a micro-spin G-25 size-exclusion column.

Dynamic single-photon emission computed tomography (SPECT)/CT acquisitions were performed immediately and 24,48 , and 72 hours following an i.v. injection of $22.0 \pm 3.6 \mathrm{MBq}$ of $\mathrm{HB}^{13}{ }^{-125} \mathrm{I}$.

Following SPECT/CT imaging, transplanted heart and recipient kidney were harvested along with major organs. Tissue samples were weighed and their radioactivity was determined by $\gamma$-well counting. The results were expressed as a percentage of injected dose per gram of tissue (\%ID/g). Recipients' kidneys were then frozen, and $40-\mu \mathrm{m}$-thick cryosections were obtained for autoradiographic imaging. Regions of interest (ROI) were drawn in order to determine graft uptake. Transplanted heart and islet graft uptake were corrected to native heart or blood activity, respectively.

Statistics. For each data set, mean \pm SD was calculated and is presented in Results in the main text. For graphical presentation of the same data sets, box plots were generated, using Prism software (version 6.01; GraphPad Software Inc.), which present the entire data set distribution. The centerline in the boxes shows the medians. Box limits indicate the 25th and 75th percentiles; whiskers indicate the minimal and maximal values. Differences between groups were evaluated (as indicated in relevant figure legends) by Mann-Whitney $U$ test, paired $t$ test, 1-way ANOVA followed by Tukey's post hoc test, or 2-way ANOVA followed by a Sidak's post hoc test, according to the size of the groups and the distribution of the variable. Islet graft survivals were compared using the log-rank test. Linear regression was used to determine the relation between 2 continuous variables (56). The differences between the groups were considered statistically significant at $P<0.05$.

Study approval. Human samples were collected at the Edouard Herriot University Hospital according to a protocol approved by the local ethical committee for clinical research (protocol no. L16-161). All subjects gave informed consent. All animal experimental procedures were approved (protocol no. ENS 2013-019) by the local ethical committee for animal research (CECCAPP, http://www.sfr-biosciences.fr/ ethique/experimentation-animale/ceccapp).

\section{Author contributions}

CCC and OT conceived and designed the experiments. CCC, EP, AB, FA, MR, PB, MA, SB, CSD, MM, JV, SDM, MNKS, AK, VM, and $\mathrm{SD}$ performed the experiments. CCC, EP, AB, FA, MR, PB, JV, VD, AN, and OT analyzed the data. LK, JPDVH, AL, TB, EM, MKT, and CG contributed to reagents. CCC and OT wrote the paper. AL, TB, MKT, AK, EM, and TD contributed to the discussion.

\section{Acknowledgments}

We thank Bernard Thorens for providing us with the $\beta$ TC-tet cell line, Shiue-Cheng Tang for sharing his expertise in islet graft imaging, Dimitri Chartoire for technical assistance, and Laurent Genestier and Helena Païdassi for fruitful scientific discussions. OT is supported by the Agence Nationale Pour la Recherche (ANR-12-PDOC-0019), the Agence de la Biomédecine (AOR-2013), the Société Francophone de Transplantation, and the Etablissement Français du Sang. CCC is supported by the Institut Hospitalo-Universitaire-Organ Protection and Replacement (IHU-OPeRa; ANR-10-IBHU-004). AK is supported by the Hospices Civils de Lyon and the Fondation du Rein. EP is supported by the Fondation Pour la Recherche Médicale (DEA20130727351). EM and OT are members of the CENTAURE Transplantation Research Network.

Address correspondence to: Olivier Thaunat, CIRI - INSERM U1111Université Lyon 1, 21 Avenue Tony Garnier, 69365 Lyon Cedex 07, France. Phone: 33.4.7211.69.28; Email: olivier.thaunat@inserm.fr.
1. Atkinson MA, Eisenbarth GS, Michels AW. Type 1 diabetes. Lancet. 2014;383(9911):69-82.

2. Miller KM, et al. Current state of type 1 diabetes treatment in the U.S.: updated data from the T1D Exchange clinic registry. Diabetes Care. 2015;38(6):971-978.

3. Kemp CB, Knight MJ, Scharp DW, Lacy PE, Ballinger WF. Transplantation of isolated pancreatic islets into the portal vein of diabetic rats. Nature. 1973;244(5416):447.

4. Scharp DW, et al. Insulin independence after islet transplantation into type I diabetic patient. Dia- betes. 1990;39(4):515-518.

5. Fioretto P, Steffes MW, Sutherland DE, Goetz FC, Mauer M. Reversal of lesions of diabetic nephropathy after pancreas transplantation. NEngl JMed.1998;339(2):69-75.

6. Kennedy WR, Navarro X, Goetz FC, Sutherland DE, Najarian JS. Effects of pancreatic transplantation on diabetic neuropathy. $N$ Engl J Med. 1990;322(15):1031-1037.

7. Barton FB, et al. Improvement in outcomes of clinical islet transplantation: 1999-2010. Diabetes Care. 2012;35(7):1436-1445
8. Sutherland DE, et al. Islet autotransplant outcomes after total pancreatectomy: a contrast to islet allograft outcomes. Transplantation. 2008;86(12):1799-1802.

9. Pouliquen E, et al. Recent advances in renal transplantation: antibody-mediated rejection takes center stage. F100OPrime Rep. 2015;7:51.

10. Sellarés J, et al. Understanding the causes of kidney transplant failure: the dominant role of antibody-mediated rejection and nonadherence. Am J Transplant. 2012;12(2):388-399.

11. Pouliquen E, et al. Anti-donor HLA antibody 
response after pancreatic islet grafting: characteristics, risk factors, and impact on graft function. Am J Transplant. 2017;17(2):462-473.

12. Chaigne $B$, et al. Immunogenicity of anti-HLA antibodies in pancreas and islet transplantation. Cell Transplant. 2016;25(11):2041-2050.

13. Brooks AM, et al. De novo donor-specific HLA antibodies are associated with rapid loss of graft function following islet transplantation in type 1 diabetes. Am J Transplant. 2015;15(12):3239-3246.

14. Piemonti L, et al. Alloantibody and autoantibody monitoring predicts islet transplantation outcome in human type 1 diabetes. Diabetes. 2013;62(5):1656-1664.

15. Ryan EA, Paty BW, Senior PA, Lakey JR, Bigam D, Shapiro AM. Beta-score: an assessment of betacell function after islet transplantation. Diabetes Care. 2005;28(2):343-347.

16. Lefaucheur C, et al. IgG donor-specific antihuman HLA antibody subclasses and kidney allograft antibody-mediated injury. J Am Soc Nephrol. 2016;27(1):293-304.

17. Sicard A, et al. Detection of C3d-binding donorspecific anti-HLA antibodies at diagnosis of humoral rejection predicts renal graft loss. JAm Soc Nephrol. 2015;26(2):457-467.

18. Loupy A, et al. Complement-binding anti-HLA antibodies and kidney-allograft survival. $\mathrm{N} \mathrm{Engl} \mathrm{J}$ Med. 2013;369(13):1215-1226.

19. Citro A, Cantarelli E, Piemonti L. Anti-inflammatory strategies to enhance islet engraftment and survival. Curr Diab Rep. 2013;13(5):733-744.

20. Merani S, Toso C, Emamaullee J, Shapiro AM. Optimal implantation site for pancreatic islet transplantation. Br JSurg. 2008;95(12):1449-1461.

21. Hirohashi T, et al. A novel pathway of chronic allograft rejection mediated by NK cells and alloantibody. Am J Transplant. 2012;12(2):313-321.

22. Jindra PT, Jin YP, Rozengurt E, Reed EF. HLA class I antibody-mediated endothelial cell proliferation via the mTOR pathway. JImmunol. 2008;180(4):2357-2366

23. Farkash EA, Colvin RB. Diagnostic challenges in chronic antibody-mediated rejection. Nat Rev Nephrol. 2012;8(5):255-257.

24. Haas M, et al. Banff 2013 meeting report: inclusion of c4d-negative antibody-mediated rejection and antibody-associated arterial lesions. Am J Transplant. 2014;14(2):272-283.

25. Nyqvist D, et al. Donor islet endothelial cells in pancreatic islet revascularization. Diabetes. 2011;60(10):2571-2577.

26. Henriksnäs J, Lau J, Zang G, Berggren PO, Köhler $\mathrm{M}$, Carlsson PO. Markedly decreased blood perfusion of pancreatic islets transplanted intraportally into the liver: disruption of islet integrity necessary for islet revascularization. Diabetes. 2012;61(3):665-673.

27. Vajkoczy P, et al. Histogenesis and ultrastructure of pancreatic islet graft microvasculature. Evidence for graft revascularization by endothelial cells of host origin. Am J Pathol.1995;146(6):1397-1405.

28. Brissova M, et al. Intraislet endothelial cells contribute to revascularization of transplanted pancreatic islets. Diabetes. 2004;53(5):1318-1325.

29. Linn T, et al. Angiogenic capacity of endothelial cells in islets of Langerhans. FASEB J. 2003;17(8):881-883.

30. Fujimoto C, Nakagawa Y, Ohara K, Takahashi H. Polyriboinosinic polyribocytidylic acid [poly(I:C)]/TLR3 signaling allows class I processing of exogenous protein and induction of HIV-specific CD8+ cytotoxic T lymphocytes. Int Immunol. 2004;16(1):55-63.

31. Mikelis CM, et al. RhoA and ROCK mediate histamine-induced vascular leakage and anaphylactic shock. Nat Commun. 2015;6:6725.

32. Cantarovich D, et al. Posttransplant donor-specific anti-HLA antibodies negatively impact pancreas transplantation outcome. Am J Transplant. 2011;11(12):2737-2746.

33. Mittal S, Page SL, Friend PJ, Sharples EJ, Fuggle SV. De novo donor-specific HLA antibodies: biomarkers of pancreas transplant failure. $\mathrm{Am} J$ Transplant. 2014;14(7):1664-1671.

34. Okitsu T, Bartlett ST, Hadley GA, Drachenberg $\mathrm{CB}$, Farney AC. Recurrent autoimmunity accelerates destruction of minor and major histoincompatible islet grafts in nonobese diabetic (NOD) mice. Am J Transplant. 2001;1(2):138-145.

35. Halloran PF, et al. Disappearance of T cell-mediated rejection despite continued antibody-mediated rejection in late kidney transplant recipients. J Am Soc Nephrol. 2015;26(7):1711-1720.

36. Huurman VA, et al. Immune responses against islet allografts during tapering of immunosuppression--a pilot study in 5 subjects. Clin Exp Immunol. 2012;169(2):190-198.

37. van Kampen CA, et al. Alloreactivity against repeated HLA mismatches of sequential islet grafts transplanted in non-uremic type 1 diabetes patients. Transplantation. 2005;80(1):118-126.

38. Heidt S, et al. A NOVel ELISPOT assay to quantify HLA-specific B cells in HLA-immunized individuals. Am J Transplant. 2012;12(6):1469-1478.

39. Breman E, et al. HLA monomers as a tool to monitor indirect allorecognition. Transplantation. 2014;97(11):1119-1127.

40. Gebel HM, Bray RA. HLA antibody detection with solid phase assays: great expectations or expectations too great? Am J Transplant. 2014;14(9):1964-1975.
41. Colvin RB, Smith RN. Antibody-mediated organ-allograft rejection. Nat Rev Immunol. 2005;5(10):807-817.

42. Wardemann H, Yurasov S, Schaefer A, Young JW, Meffre E, Nussenzweig MC. Predominant autoantibody production by early human B cell precursors. Science. 2003;301(5638):1374-1377.

43. Kratz A, Campos-Neto A, Hanson MS, Ruddle NH. Chronic inflammation caused by lymphotoxin is lymphoid neogenesis. J Exp Med. 1996;183(4):1461-1472.

44. Thaunat $\mathrm{O}$, et al. Lymphoid neogenesis in chronic rejection: evidence for a local humoral alloimmune response. Proc Natl Acad Sci U S A 2005;102(41):14723-14728.

45. Thaunat O, et al. Chronic rejection triggers the development of an aggressive intragraft immune response through recapitulation of lymphoid organogenesis. JImmunol. 2010;185(1):717-728.

46. Thaunat O, et al. A stepwise breakdown of B-cell tolerance occurs within renal allografts during chronic rejection. Kidney Int. 2012;81(2):207-219.

47. Thaunat $\mathrm{O}$, et al. Immune responses elicited in tertiary lymphoid tissues display distinctive features. PLOS ONE. 2010;5(6):e11398.

48. Shiba Y, et al. Allogeneic transplantation of iPS cell-derived cardiomyocytes regenerates primate hearts. Nature. 2016;538(7625):388-391

49. Thaunat $O$, et al. B cell survival in intragraft tertiary lymphoid organs after rituximab therapy. Transplantation. 2008;85(11):1648-1653.

50. Clark RA, et al. Skin effector memory T cells do not recirculate and provide immune protection in alemtuzumab-treated CTCL patients. Sci Transl Med. 2012;4(117):117ra7.

51. Iijima N, Iwasaki A. Access of protective antiviral antibody to neuronal tissues requires $\mathrm{CD} 4 \mathrm{~T}$-cell help. Nature. 2016;533(7604):552-556.

52. Chen ZH. A technique of cervical heterotopic heart transplantation in mice. Transplantation. 1991;52(6):1099-1101.

53. Dimitrov JD, Lacroix-Desmazes S, Kaveri SV. Important parameters for evaluation of antibody avidity by immunosorbent assay. Anal Biochem. 2011;418(1):149-151.

54. Efrat S, Fusco-DeMane D, Lemberg H, al Emran $\mathrm{O}$, Wang X. Conditional transformation of a pancreatic beta-cell line derived from transgenic mice expressing a tetracycline-regulated oncogene. Proc Natl Acad Sci U S A. 1995;92(8):3576-3580.

55. Duong Van Huyen JP, et al. GDF15 triggers homeostatic proliferation of acid-secreting collecting duct cells. J Am Soc Nephrol. 2008;19(10):1965-1974.

56. Altman N, Krzywinski M. Simple linear regression. Nat Methods. 2015;12(11):999-1000. 